\title{
Phase transitions in Virology
}

\author{
Ricard Solé, ${ }^{1,2,3}$ Josep Sardanyés, ${ }^{4,5}$ and Santiago F. Elena ${ }^{6,3}$ \\ ${ }^{1}$ ICREA-Complex Systems Lab, UPF-PRBB, Dr. Aiguader 80, 08003 Barcelona, Spain. \\ ${ }^{2}$ Institut de Biologia Evolutiva, CSIC-Universitat Pompeu Fabra, Passeig Maritim de la Barceloneta 37, 08003 Barcelona, Spain. \\ ${ }^{3}$ Santa Fe Institute, 1399 Hyde Park Road, Santa Fe NM 87501, USA. \\ ${ }^{4}$ Centre de Recerca Matemàtica (CRM); and Barcelona Graduate School of Mathematics \\ (BGSMath). Edifici C, campus de Bellaterra, Cerdanyola del Vallès, 08193 Barcelona, Spain. \\ ${ }^{5}$ Dynamical Systems and Computational Virology, Associated Unit \\ Instituto de Biología Integrativa de Sistemas (I'SysBio) - CRM \\ ${ }^{6}$ Evolutionary Systems Virology Lab, I'SysBio, CSIC-Universitat de \\ València, Catedrático Agustín Escardino 9, Paterna, 46980 Valencia, Spain.
}

\begin{abstract}
Viruses have stablished symbiotic relationships with almost every other living organism on Earth and at all levels of biological organization, from other viruses up to entire ecosystems. In most cases, peacefully coexisting with their hosts, but in most relevant cases, parasitizing them and inducing diseases. Viruses are playing an essential role in shaping the eco-evolutionary dynamics of their hosts, and also have been involved in some of the major evolutionary innovations either by working as vectors of genetic information or by being themselves coopted by the host into their genomes. Viruses can be studied at different levels of biological organization, from the molecular mechanisms of genome replication, gene expression and encapsidation to global pandemics. All these levels are different and yet connected through the presence of threshold conditions allowing for the formation of a capsid, the loss of genetic information or epidemic spreading. These thresholds, as it occurs with temperatures separating phases in a liquid, define sharp qualitative types of behavior. These phase transitions are very well known in physics. They have been studied by means of simple, but powerful models able to capture their essential properties, allowing to understand them. Can the physics of phase transitions be an inspiration for our understanding of viral dynamics at different scales? Here we review the best known examples of transition phenomena in virology and their simplest mathematical modeling approaches. We suggest that the advantages of abstract, simplified pictures used in physics are also the key to properly understand the origins and evolution of complexity in viruses. By means of several examples, we explore this multilevel landscape and how minimal models provide deep insights into a diverse array of problems. The relevance of these transitions in connecting dynamical patterns across levels and their evolutionary and clinical implications are outlined.
\end{abstract}

Keywords: Epidemics, molecular replicators, mutation, multi-level selection, quasispecies, recombination, self-assembly, phase transitions, virus evolutionary dynamics

\section{INTRODUCTION}

Researchers from different disciplines tend to study viruses at their favorite level of biological organization (Fig. 1). At the lowest scale of biological complexity, molecular virologists carefully characterize the mechanisms of viral replication and pathogenesis, the molecular interactions between viral proteins and among viral and host proteins, and how cells respond to infection by triggering diverse defense mechanisms. At the highest scale, ecologists and epidemiologists describe the incidence of viruses in their reservoir and potential novel hosts, and their spread among host populations and at the global level, including their impact on nutrient cycling. At each scale, what is considered as the 'host' is different: single cells or tissues for molecular virologists, individuals for clinical virologists and plant pathologists, populations of hosts or even entire ecosystems for ecologists and epidemiologists. Likewise, the 'virus' unit upon which selection operates also differs among the levels of biological organization being studied: mutant swarms within individuals, infected individuals in ecological studies or viral lineages in phylogeography and epidemiological studies. But the ultimate host for any virus is always an individual cell. It is inside the cell wherein the virus expresses its genes, kidnaps the cellular resources and manipulate structures to build the replication factories. Viral proteins and RNAs interact with a large number of cellular factors in order to block cell defenses, to ensure reproduction, encapsidation and spread to neighboring cells. From there to colonize other tissues and organs, and from there to be transmitted to other susceptible hosts, to spread in the host populations and, ultimately, to reach diverse ecosystems.

Different selective pressures operate on viruses at each of the above levels. For example, whereas at the cellular and within-organism levels fast replication may provide competitive advantage to one strain over another, at the population level such strategy may not be optimal, as it jeopardizes transmission between hosts by immobilizing the infected host and thus effectively reducing the rate of transmission (Doumayrou et al. 2012). Another remarkable example of seemingly opposed observations: population-level human immunodeficiency virus 
type 1 (HIV-1) phylogenies are mainly shaped by selective neutral epidemiological processes, implying that genealogy-based population genetic inferences can be useful to study the HIV-1 epidemic history and dating events (Lemey et al. 2006). In sharp contrast, HIV-1 phylogenies reconstructed from within-host sequences indicate the action of strong selective pressures imposed by the heterogeneity of cell types in which the virus can be replicated (Lemey et al. 2006). Indeed, the existence of within-host reservoirs of latently infected CD4 ${ }^{+} \mathrm{T}$ cells produces a delay in the evolutionary dynamics within single hosts. These delays can fundamentally change the dynamics of the virus transmission between individuals and, hence, have an impact at the epidemiological scale (Doekes et al. 2017). Therefore, while the general connection between the infection dynamics within a host and the population-level transmission dynamics of viruses is widely acknowledged, a comprehensive and quantitative understanding that would allow full integration of the two scales is still lacking.

There have been attempts to model multi-scale selection for viruses. At most, studies have tried to model between-host transmissions as a function of within-host replication parameters (Coombs et al. 2007; Mideo et al. 2008; Metzger et al. 2011; Feng et al. 2012; Schole et al. 2013; Shin and MacCarthy 2016; Doekes et al. 2017; Dorratoltaj et al. 2017), or tissue/organ colonization as an extension of within-cell replication events and interaction with host factors (Sardanyés and Elena 2011; Heldt et al. 2013; Kumberger et al. 2016). Unfortunately, the former models usually ignore the inherent within-host complexity, while the latter never extend beyond the individual tissue or single host. Gong et al. (2015) identified seven challenges for developing multi-scale models of virus evolution:

1. Lack of models and data to elucidate the processes underlying transmission probabilities and bottlenecks. From the donor host side, how does infectiousness depend on virus load? From the recipient host side, initial infection depends on the dose (bottleneck), route of transmission, time of exposure, etc. Stochastic and spatial invasion models will offer insights if feed with the appropriate empirical data.

2. Heterogeneity within a single host. Many of the existing within-host models describe the host as a single population of target cells without any structure. This is obviously not the case and heterogeneity in cell type, spatial structure, susceptibility and immune response will all play important roles in shaping infection dynamics.

3. Fitness landscapes may be highly dynamic and variable between host types. Very little is known about the topography of the fitness landscapes into which viral populations evolve. Evidences suggest they may be rugged but also contain some degree

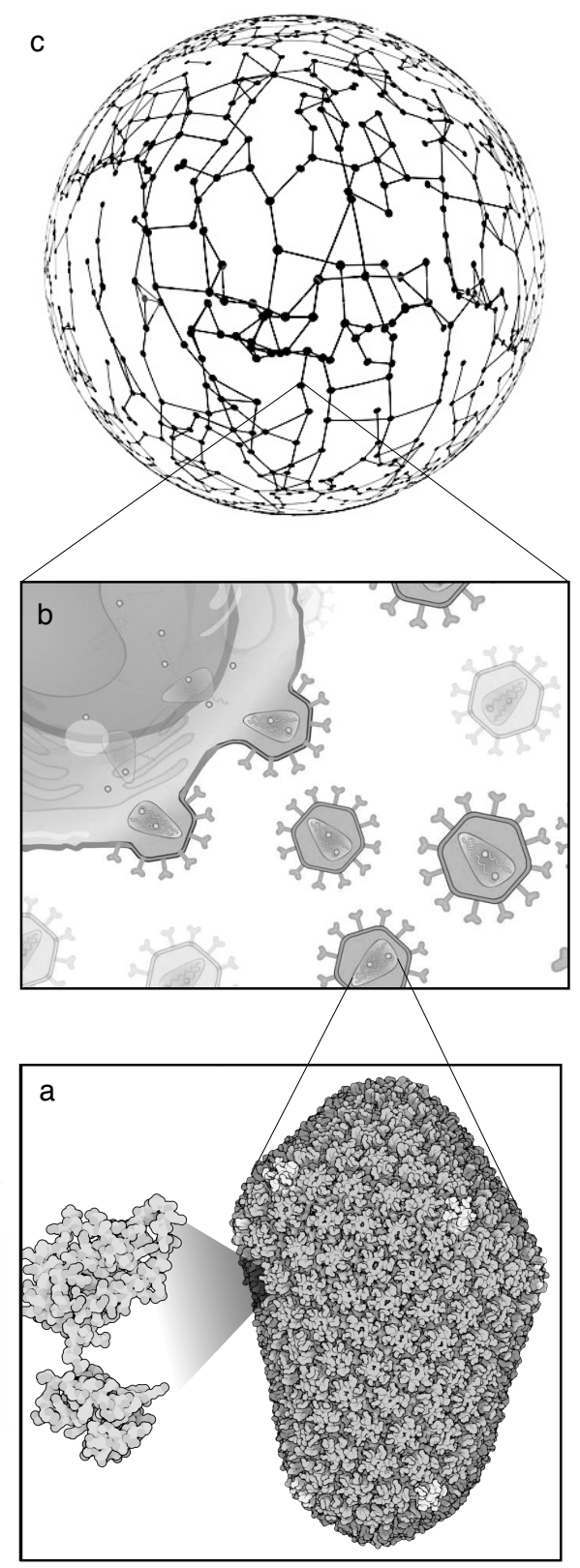

FIG. 1: The multiple levels of virus dynamics. Here only three key examples are displayed, namely (a) molecular assembly (b) virus-cell infection, and (c) social network of individual hosts, represented as a graph with links indicating potential transmissions. Between these levels, other intermediate scales such as tissue/organ levels need to be considered.

of neutrality (Kouyos et al. 2012; Lalić and Elena 2015). Landscape's topography may be strongly affected by the cell types and immune responses, with epistasis (Elena et al. 2010) and antagonistic pleiotropy being essential components (Cervera et al. 2016). Indeed, it has been recently suggested that the dominant view among biologists of a static fitness landscape as a succession of valleys and peaks may be misleading to explain many ob- 
servations in virus evolution. Instead, it has been suggested that an adaptive multiscape (Catalán et al. 2017), or a time-fluctuating adaptive seascape (Mustonen and Lässig 2009), may provide a much better representation.

4. Current models do not easily incorporate highthroughput next-generation sequencing (NGS) data. Empirical studies have demonstrated that during acute and chronic infections, RNA viruses generate massive amounts of genetic variability (Domingo et al. 2012). In some cases, this genetic diversity is transmitted, in some other not. NGS provides valuable information to assess the size of bottlenecks and the spread of resistance variants. Furthermore, quantitative methods have been established at the epidemiological level, or using global level phylogeography analyses, typically based on consensus sequences, but there seem to be no well-established methods for analysis of NGS data at this level, thus missing the opportunity to link within- and between-individual diversity with epidemiological processes.

5. Ignoring superinfection ${ }^{a}$ has greatly simplified modeling efforts. However, it is not clear when this approximation should be valid. Superinfection is known to be important in many viruses, e.g. HIV1 , increasing viral load and hastening progression to AIDS (Korenromp et al. 2009).

6. The distinction between within- and between-host dynamics can be easily made. However, the fact that both of these scales involve further nested levels has been often neglected. While multi-scale models at the population level are common, these models ignore the within-host components. This lack of integration of within-host levels of complexity, so far, resulted from the lack of information in vivo. Fortunately, this limitation is being overcome with NGS and the development of models that take into account cellular processes (e.g., Loverdo et al. 2012).

7. What approaches should be used to link processes across scales? modeling multi-scale processes in full mechanistic detail, or even simulating such models, is unrealistic. One possibility is to come up with ways of extracting the essential features of lower-scale models to embed them into higher-scale models efficiently (Mideo et al. 2008). An approach that has been successfully taken is to separate timescales, which essentially separates models that may be used at different scales. Following

a Superinfection refers to the process in which an already infected host becomes secondarily infected by a different virus or a different strain of the same virus. this philosophy, Park et al. (2013) used Markov chain modeling at the within-host level embedded into a stochastic branching process for betweenhost transmission. The challenge is to develop better methods for incorporating multiple scales into a single framework.

As complex adaptive dynamical systems, viruses experience critical phase transitions at different levels of organization (Solé and Elena, 2019). These critical transitions involve a sudden change in the dynamical behavior or the internal structure of the system. At the lowest molecular level, the concept of critical points at phase transitions have been used to describe process such as the assembly and disassembly of viral particles (Dharmavaram et al. 2017), the error threshold associated to the highly mutagenic replication of viral genomes (Eigen 1971), the spread of perturbations across host proteinprotein and regulatory networks induced by viral factors (Bosque et al. 2014), and the agglomeration of viral particles to form pseudo-crystal structures which may operate as units of selection (Cuevas et al. 2017). At the individual patient level, coordinated changes in expression levels of host proteins linked into functional modules may act as early-warning signals for the critical transition between healthy and disease stages during infection (Liu et al. 2012). Finally, at the epidemiological level, spread of a viral disease in a scale-free network formed by connected populations of susceptible host show complex dynamical behavior and phase transitions from nonepidemic to epidemic stages associated with the existence of highly connected hubs (Barthélemy et al. 2005; Balcan and Vespignani 2011). So far, models connecting phase transitions at different levels have not been proposed. Likewise, no model has been brought forward to explore whether transitions at one level may results from the dynamical properties of the levels below, especially whether the role of natural selection at each level may interplay with criticality.

In this article, we promote the idea that phase transitions should be considered as a unifying principle across the different scales of virus complexity. In the following sections, we will illustrate this idea by presenting a number of well-characterized examples of phase transitions in virology. All selected examples involve transitions between different, well-defined phases. These include: selfassembly of viral particles, intra-host infection dynamic, the existence of epidemic thresholds, the triggering of large-scale pandemics on highly connected networks, and the evolution of multipartite viruses as a case of mutualistic interactions between molecular replicators. In addition, we will describe three more examples that fit within the more general class of information and diversity thresholds: the error catastrophe at increasing mutation rates, the two-sides of recombination in purging deleterious variation or in driving towards extinction, and the antigenic diversity threshold in the set point of HIV-1 progression. In all examples, we will provide a simplified mathematical description of the system and show the ex- 
a

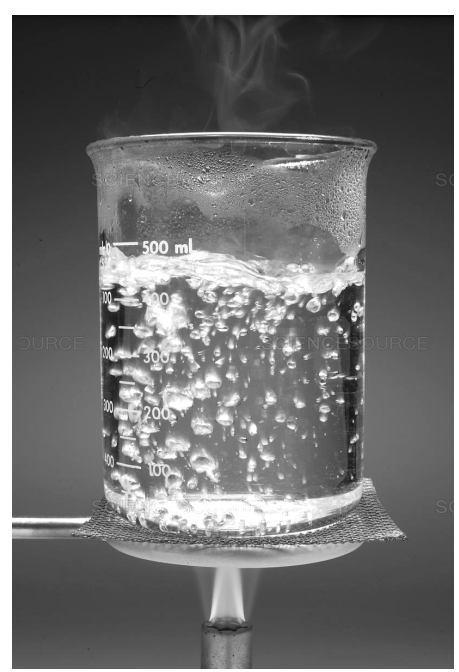

C

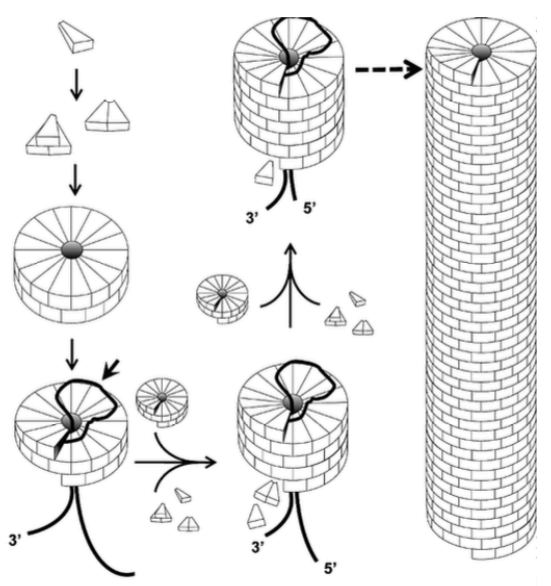

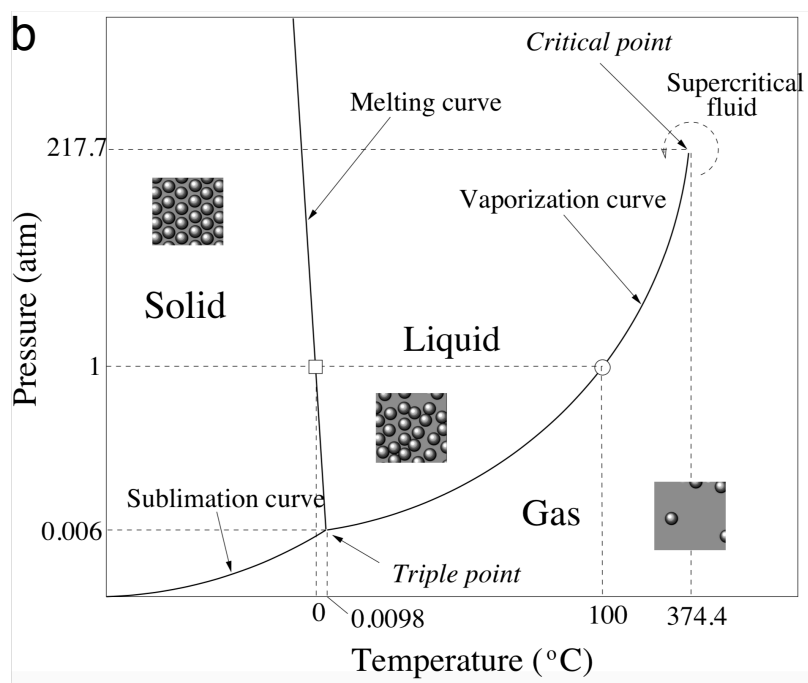

d

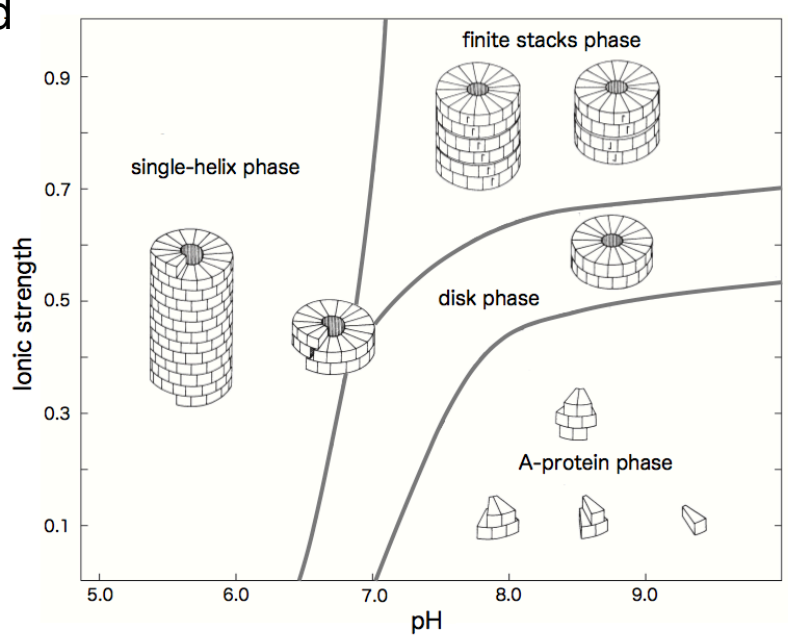

FIG. 2: Phase transitions in both physics and virus dynamics involve marked qualitative changes. An example is (a) the boiling of water, where the two phases (liquid and steam) coexist. For different values of temperature and pressure, a phase diagram (b) reveals three basic phases are separated by well-defined critical curves. The molecular organization of viral capsids is also sharply affected by key parameters. An example is the formation of TMV that takes place by self-assembly of coat proteins (c) leading to long rods. But such process and the final nature (phase) of the self-assembly process is sharply controlled by physicochemical parameters, such as $\mathrm{pH}$ or ionic strength. This pair of parameters allow displaying a phase diagram (d) that also exhibits transition lines between different configurations.

istence of these critical organization thresholds that can be clearly identified by means of the so-called bifurcations. Readers less interested in mathematical details can jump over the next section without losing the main messages.

\section{PHASE TRANSITIONS: SIMPLE MODELS EXPLAIN COMPLEX CHANGES}

Phase transitions are introduced in this paper with the aim of providing a proper framework to understand the nature of change in virology. By change we refer to the qualitative dynamical and structural shifts of organization of complex systems. The term phase transition was coined to describe the sudden shift between states of matter, but has been since then generalized to many other domains (Solé, 2011). An example is the phase change from ice to liquid water or from water to steam, as shown in Fig. 2a . Each phase is defined by some state that is uniform within its domain in the phase diagram, which essentially tells us which phases are stable under what conditions. Such properties can rapidly change close to the boundaries between phases. Figure $2 \mathrm{~b}$ shows a classical example, namely the transition from liquid water to steam. Within a given phase, no major differences can be seen beyond smooth quantitative changes. As an example, when water is heated from $2{ }^{\circ} \mathrm{C}$ to $25{ }^{\circ} \mathrm{C}$ the same state (liquid) is observed and its density only 
changes slightly (by just $2 \%$ ) while it decreases 1600fold as boiling temperature is reached. In other words, a slight change close to the transition point drives a very large, abrupt change in density. Such abrupt transition is termed "first-order" and is also observed in the melting of ice. This occurs also in other diverse systems, such as polymers and other materials experiencing qualitative structural re-arrangements (Dill and Bromberg 2012), abrupt climate change (see Solé 2011 and references therein) or ant colonies exploring their environments (Beekman et al. 2001; Piñero and Solé 2019). In all these systems it is possible to define a phase diagram showing the parameter values at which the different states (phases) are found. Now, instead of temperature and pressure, the axes can include, for example, molecular concentrations or strength of ionic forces, which affect the kinds of capsid assembly for the tobacco mosaic virus (TMV) (Fig. 2c-d). The process of virus assembly requires the formation of rods (Fig. 2c) which is affected by key external parameters defining a space of shapes (Fig. 2d). The structures shown here are the dominant ones in each phase (Klugg 2010).

A different class of transition, known as a second-order phase transition, involves a qualitative change as well but this change occurs in a smooth manner as parameters vary. This is the case for example in the behavior of ferromagnetic materials: when a magnet is heated, it initially maintains its magnetization, which decays until it vanishes altogether at a critical temperature $T_{c}$. In these transitions, remarkably rich behavior can be observed, including both very high variance in both structural and dynamical traits. wide fluctuations in the measured macroscopic properties (such as magnetization) are observed as we approach criticality (the phase transition point) where the variance diverges. For this example, we have a microscopic description that allowed to formulate a simple model capable of capturing most relevant features of the real transition. This is the so called Ising model or, as physicist Nigel Goldenfeld calls it, "the Drosophila model of statistical mechanics".

A magnet can be imagined as a lattice of units (atoms) each one describable as some kind of magnet itself, with two possible orientations (or "spins"): up and down. Mathematically, that means that we have a collection of $N$ units whose states are indicated as $S_{k}$, located on a geometric grid and having two possible states, say $S_{k}=-1$ and $S_{k}=+1$. From quantum mechanical arguments, it is known that the lowest energy state (and thus the more likely) is the one where nearest atoms have the same spin states (with some probability). If the two types of spins are indicated as +1 and -1 , the difference between the number of up spins $\left(N_{+}\right)$and down spins $\left(N_{-}\right)$, defines the global magnetization $M$, i.e. $M=N_{+}-N_{-}$. This value gives the capacity of the magnet to attract other ferrous materials. As temperature grows, the coupling between spins becomes less and less strong. If temperature $T$ is below a critical value, labeled $T_{c}$, interactions among nearest spins would favor a dominant direction.
Assuming that the system started from a random initial condition, the outcome of spin-spin interactions is one of two possible macroscopic ordered states: either a majority of up spins or the alternative dominated by down spins. However, if $T>T_{c}$, disorder wins, and spins essentially behave randomly. This is thus a transition between two equally likely ordered states where $M \neq 0$ and a disordered phase with $M=0$. Within each of the two phases, all measurable properties change smoothly.

The previous observations can be captured using a microscopic model (the so called Ising model) that involves defining an energy function $H$ given by the sum (Christensen and Moloney, 2005)

$$
H=-\frac{1}{2} \sum_{\langle i, k\rangle} J S_{k} S_{i},
$$

where $J$ is a coupling constant that weights the strength of spin-spin interactions. The bracket $\langle i, k\rangle$ indicates that the pairs are restricted to nearest neighbors. As defined, we can see that energy decreases (as it occurs with any spontaneous phenomenon) when $S_{i} S_{k}=1$, i.e. when both spins are oriented in the same direction. Along with this energy function, a set of rules is needed to introduce changes into the state of the units as they interact under a given temperature. In a nutshell, we need probabilities of transition $P\left[S_{i} \rightarrow 1-S_{i}\right]$ for every chosen unit $S_{i}$ between the two possible states, that include on one hand the effect of temperature (the higher, the weaker the interaction) and, on the other hand, the set of neighbors of the chosen unit. The larger the fraction of neighbors pointing in the same direction, the more likely that our unit will follow them. It can be shown (Solé 2011) that one simple way of implementing this probability is:

$$
P\left[S_{i} \rightarrow 1-S_{i}\right]=\frac{1}{1+\exp (\Delta H / k T)},
$$

where $\Delta H$ indicates the change in energy associated to the spin flip. It can be easily shown that this transition probability properly behaves as expected: it will be close to 0.5 (coin toss) for high temperatures while will be close to one at low temperature when the energy change is favorable, i.e. when $\Delta H<0$ (see also chapter two in Solé and Goodwin, 2001).

A specially important consequence of the Ising model was the realization that it was able to explain not just the presence of phase transitions. When analyzing the quantitative properties of this model close to critical points, it turned out that they predicted with enormous precision the experimental observations. In other words, in order to accurately explain the experimental data, extremely simple models with the minimal description of units and their interactions turned to be enough. Actually, very different systems undergoing phase transitions were shown to respond to exactly the same class of minimal model. Such universality pervades the theory of critical phenomena (Stanley et al. 1996, Kadanoff 2000, Goldenfeld 2018) and has been used in multiple scenarios, from cosmology to social and economic systems. 
A full analysis of these Ising-like models would require going deeper into statistical physics methods (Goldenfeld 2018) but it can be shown that on a coarse-grained approximation, the so called mean field ${ }^{b}$ Ising-like models follow the simple differential equation (Christensen and Moloney, 2005; Solé 2011)

$$
\frac{d M}{d t}=f_{T}(M)=\left(T_{c}-T\right) M-M^{3}
$$

where $M$ is the magnetisation but would indicate, for example, the average values of concentration or populations levels. This is the kind of mathematical approximation followed in this paper, where we will also ignore most details of the interactions among different components, as well as stochastic and spatial effects (Stollenwerk and Jansen 2011) which require mathematical developments beyond the scope of our contribution. The last equation does represent fairly well what takes place when a magnet experiences the phase transitions. The relevant states are defined by the so called fixed points of the model, namely those $M^{*}$ such that

$$
\left(\frac{d M}{d t}\right)_{M=M^{*}}=0
$$

in other words, those $M$ such that the the right-hand side of (3) is zero. This gives three solutions: (a) the zeromagnetization state $M^{*}=0$ which would be associated to the disordered state (equal average numbers of up and down spins) and two additional states

$$
M_{ \pm}^{*}= \pm \sqrt{T_{c}-T}
$$

which only exist when $T<T_{c}$, i. e. at the low- $T$ phase. It can be shown (Strogatz 1994) that each of these points will be stable if the sign of

$$
\lambda\left(M^{*}\right)=\left(\frac{d f_{T}(M)}{d T}\right)_{M=M^{*}}
$$

is negative. As an example, $M^{*}=0$ will be stable (and that. will be the observable state) when $T>T_{c}$ while the two others will be stable if the opposite condition $\left(T<T_{c}\right)$ holds. Using this class of model the location of $T_{c}$ and other quantities will deviate from the spatial system, when only nearest neighbors interact. However, this is a rather limited context that can be ignored here. In most cases, these models are enough to capture the presence and implications of transition phenomena.

An additional message that emerges from the success of simple models is that even apparently too complicated systems can be properly approached by highly simplified

\footnotetext{
${ }^{b}$ Mean field models are a very powerful tool in statistical physics. They ignore local, spatial correlations among the interacting entities assuming the system is perfectly mixed. This assumption usually allows to obtain dynamical equations.
}

dynamical pictures. This is in fact at the root of the success of mathematical models and, to a large extent, the success of well established disciplines like the physics of collective phenomena and, in a more general sense, the theory of complex systems. Intuition tells us that a complicated system (almost any real system we would handle) would require a proportional degree of detail and complication. If that were true, models would be seldom useful to understand reality, since they would become too complicated to be understood. The good news is that, for most complex nonlinear systems, there are a few key variables that play a major role in controlling the dynamics of different phases of a system as well as the presence of transitions between them (Haken 1975; Nicolis 1995).

In this paper we present several well-defined, simple models for a diverse range of problems in virology. Although models incorporating microscopic dynamics have been developed, our approach is based on mean field approximations where only the population-level dynamics is taken into account. This means that we consider the average features of interactions and use them to construct population-level descriptions. This allows to show the general relevance of the concept of phase transition, its conditions for occurrence and relevance, avoiding a more technical and lengthy presentation of each case study.

\section{A. Self-assembly of viral particles}

As a first example of phase transition at the molecular scale, let us consider the self-assembly of viral capsids. This process depends on energy-driven physical forces. Self-assembly results from cooperative interactions under the laws of physics and is a crucial component of emergent dynamics (Whitesides and Grzybowski 2002; Freitas and Merkle 2004; Mouritsen 2005; Solé 2009). We can treat viral assembly as an energy minimization process (Hagan and Chandler 2006, Higks and Henely 2006, Rossman and Rao 2012, Perlmuter and Hagan 2015). As pointed out by Zlotnick (2004), understanding the physics of viral self-assembly requires a proper knowledge of the requirements of viral stability and how it is regulated. In this section we consider the problem of how key parameters such as the concentration of capsomers can act as a control parameter for a phase transition separating a set of disconnected units from a fully formed capsid. How such model can be formulated and what are its consequences?

Several studies have used well-defined physical models of capsomer structure and interactions, usually based on different extensions of well-known reaction kinetic models including self-assembly and polymerization (Kushner 1969) and, in particular, physical models of viral assembly based on the minimization of energy functions (Bruinsma et al. 2003; Zandi et al. 2004; Hagan 2014). Among the most interesting results offered by these modelling approaches, when applied to spherical viruses, is 

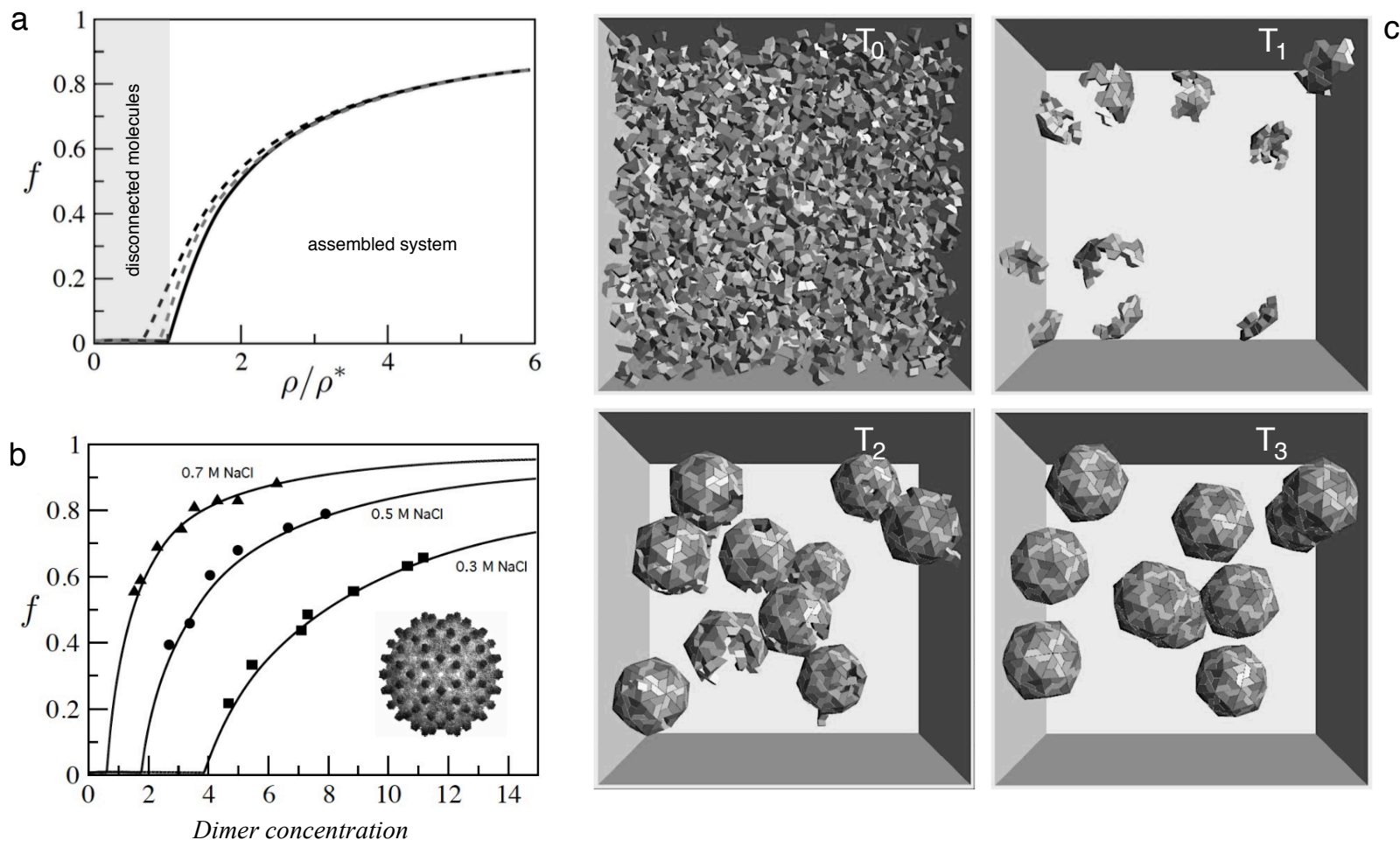

FIG. 3: Second-order phase transition in the capsid assembly process. (a) Predicted theoretical result $f_{c}\left(\rho_{T}\right)=1-\left(\rho^{*} / \rho_{T}\right)$ for (from left to right) $N=12,60$ and 1000, respectively. $f(\rho)$ represents the fraction of capsids present in a given cell as a function of the capsomer concentration $(\rho)$. An experimental test of this theoretical result is shown in panel (b) using empty capsids of hepatitis B virus (inset) under different dimer subunit and salt concentrations (adapted from Hagan (2014)). In (c) several snapshots from a spatial simulation of capsid self-assembly are shown (modified from Rapaport 2010). They show an early state $\left(T_{0}\right)$ and several intermediate states where only shells and well formed capsids are shown (the units not forming these structures are not shown in $T_{1}, T_{2}, T_{3}$ for proper visualization of the larger structures)

the finding that the limited repertoire of possible icosahedral 'solutions' corresponds to the minima of an energy landscape (Bruinsma et al. 2003) thus showing that physics pervades the constraints associated to the universe of viral forms. One important side effect of this result is the explanation for the discrete nature of possible icosahedral viruses and their 'mathematical' nature (Stewart 1999).

Let us now explore the simplest picture of self-assembly processes based in a kinetic model leading to a dynamical pattern of aggregation characterized by the presence of a second-order phase transition behavior (Dill and Bromberg 2011). This can be illustrated by a minimal model where a set of $n$ capsomer aggregate to form an entire capsid. If we indicate by $A_{1}$ single building capsomers and as $A_{n}$ the full assembled capsid, the reaction for the whole process could be easily described by

$$
n A_{1} \stackrel{K}{\longrightarrow} A_{n}
$$

where $K=\left[A_{n}\right] /\left[A_{1}\right]^{n}$ is the equilibrium constant. If we indicate by $C_{0}$ the initial concentration of $A_{1}$, the reaction kinetics for $A_{1}$ would be described by a nonlinear equation $d A_{1} / d t=-K A_{1}^{n}$ whose solution is given by

$$
A_{1}(t)=\left[\frac{C_{0}}{1+K C_{0}(n-1) t}\right]^{\frac{1}{n-1}},
$$

displaying two markedly different behaviors as a function of $K$. This can be shown by looking at the fraction $\nu(x)$ of components associated to assembled aggregates as a function of $K$, where $x=\left[A_{1}\right]$. Since $\left[A_{1}\right]+n\left[A_{n}\right]$ is the total number of capsomers and $\left[A_{1}\right]+\left[A_{n}\right]$ the total number of 'objects'

$$
\nu(x)=\frac{1+n K x^{n-1}}{1+K x^{n-1}},
$$

which exhibits a sharp transition close to a critical value given by

$$
x_{c}=K^{-1 /(n-1)} .
$$

This cooperative behavior indicates that, once a critical capsomer concentration $x_{c}$ is reached, the system experiences a rapid and irreversible transition into large structures with a characteristic size. This occurs in a thermodynamically favored direction, and thus the resulting assemblies are highly stable structures. In a 
chemical system formed by inert molecules, self-assembly takes place by an energy-minimization process (that is captured in the irreversible reaction described above) eventually ending up in stable assemblies. Continuous translation of the virus' capsid protein gene(s) into capsomers creates the conditions for this transition as the concentration of capsomers in the cell cytoplasm increases until the $x_{c}$ required for self-assembly is reached.

The specific application of this approach to the selfassembly of viral capsids can be used to obtain an expression for the fraction of capsids $f(\rho)$ present in a given system (such as the cytoplasm) as a function of the capsomer concentration ( $\rho$ ) assuming that (as before) we neglect all molecular intermediates except free capsomers (Hagan 2014). If the total concentration of capsid units $\rho_{T}$ is given by: $\rho_{T}=\rho_{1}+N \rho_{N}$, where $N$ is the number of capsomers necessary to build a capsid, and $\rho_{1}$ and $\rho_{N}$ the densities of single capsomers and whole capsids, respectively, one can define the fraction of capsomers already forming part of capsids using

$$
f_{c}=\frac{N \rho_{N}}{\rho_{T}} .
$$

A critical concentration $\rho^{*}$ exists such that a phase transition occurs, separating a sub-critical phase with essentially no self-assembly of viral particles, i.e.,

$$
f_{c}\left(\rho_{T}\right) \approx\left(\frac{\rho_{T}}{\rho^{*}}\right)^{N}
$$

for $\rho_{T} \ll \rho^{*}$, from a second phase in which virus capsids form, and $f$ reads

$$
f_{c}\left(\rho_{T}\right)=1-\frac{\rho^{*}}{\rho_{T}}
$$

when $\rho_{T} \gg \rho^{*}$. The phase transition curves predicted from the model are displayed in Fig. 3a, using three different values of $N$. The results of an experimental test of this model are shown in Fig. 3b, where different concentrations of capsomers have been used under variable salt concentrations enhancing the self-assembling process (see Hagan (2014) for details).

The study of the microscopic processes underlying viral assembly has been a very active area where the use of both kinetic and thermodynamic (physical) models has been very successful. In particular, molecular dynamic models provided the basis for describing the details of such self-assembly using energy functions that capture the nature of the underlying potentials (Rapaport 2010, 2014). In Fig. 3c we display an example of the simulation outcome of this process, where the units have a three-dimensional geometry and assembly follows several intermediate steps, involving for example the formation of shells. More sophisticated models allow to study the interaction between genomes and capsomers as the full viral particle is formed (Permuter et al 2013).

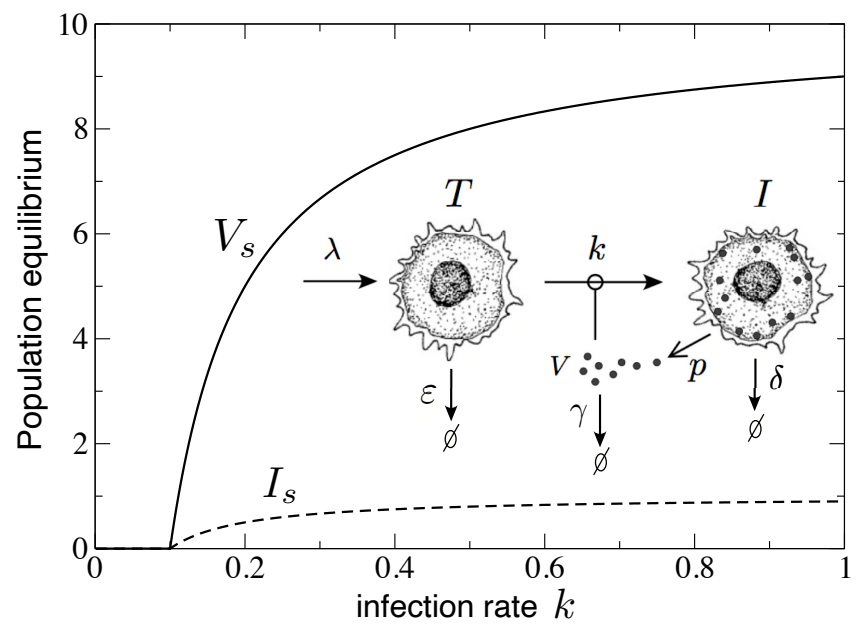

FIG. 4: Simple models and complex interactions. Secondorder phase transition showing how the equilibrium values of the virus population $\left(V_{s}\right.$, solid line) and infected cells $\left(I_{s}\right.$, dashed line) diminish at decreasing the infection rate $k$. The inset displays a schematic diagram of the model, with the interactions between the susceptible cells $(T)$ and viruses $(V)$ (drawings by R. Solé). See Eqs. (13)-(15) for the mathematical description.

\section{B. Intrahost infection dynamics}

When a model of cell-virus interactions is to be build, population biologists usually consider the number of individuals in each two species (say $X$ and $Y$ for cells and virus, respectively) as the key variables. Interaction, growth and mortality parameters are then introduced and the main problem is how to properly express the functional relations associated to all these processes (Case 2000). The reactions required to represent this Lotka-Volterra type model (May 1973; Gotelli 1988) are represented schematically in the inset of Fig. 4. One could say that this is too simple to represent the true complexity of a natural virus-cell system, but the truth is that this toy model accounts for one particularly relevant property of these systems, namely the presence of cycles, which are a consequence of the internal dynamics of the system. Instead of being driven by some external driver, the nonlinearities of the model fully account for the emergence of oscillatory behaviour. This oscillatory regimes have been recently identified in mathematical models incorporating further complexity in infection processes (Nurtay et al. 2018).

Consider now the problem of a minimal model of viral infection (Fig. 4) where three variables are at least needed: the number of target cells $(T)$, the number of infected cells $(I)$ and the amount of viruses $(V)$. This model has been studied in detail elsewhere (Perelson and Nelson 1999; Statford et al. 2000; Perelson 2002; Nowak et al. 1996) and assumes that a population of cells is generated at some constant rate $\lambda$. These cells are the target of a virus that infects them at some rate $k$ and 
this process generates infected cells, which produce virus particles at some rate $p$ (the burst size). All the three populations decay with given rates (indicated as $\varepsilon, \delta$ and $\gamma$ in the inset of Fig. 4).

The set of interactions also involves pairwise 'reactions', but in this case viruses transform their host (turning $T$ cells into infected $I$ cells) into a different type of 'particle' and moreover they are produced by the infected cell population $I$ at a constant rate. Additionally, the model assumes a completely mixed system where encounters between viruses and cells occur at random with homogeneous rates. This is of course another simplification: susceptible cells are usually strongly structure in space forming tissues, the model considers no evolution of the viral component, which of course is also a very strong assumption.

The following set of equations describes the basic dynamics outlined in the inset of Fig. 4:

$$
\begin{aligned}
\frac{d T}{d t} & =\lambda-\varepsilon T-k V T \\
\frac{d I}{d t} & =k V T-\delta I \\
\frac{d V}{d t} & =p I-\gamma V .
\end{aligned}
$$

This model gives intuition concerning the potential outcomes of the infection dynamics. It has two alternative equilibrium points (steady states), obtained from $d T / d t=d I / d t=d V / d t=0$. The first corresponds to the virus-free system, and is given by $S_{0}=(\lambda / \varepsilon, 0,0)$, whereas the second equilibrium point provides the steady state $S_{1}=\left(T_{s}, I_{s}, V_{s}\right)$ where we have a steady infected population

$$
I_{s}=\frac{1}{\delta}\left(1-\frac{\varepsilon \gamma \delta}{p k}\right)
$$

and the equilibrium value for the virus population given by

$$
V_{s}=\frac{p}{\delta \gamma}-\frac{\varepsilon}{k}
$$

The last expression gives a critical condition for the virus to persist: since we need $V_{s}>0$ to meet this condition, we have $p / \delta \gamma>\varepsilon / k$ or, by rearranging terms, if

$$
R_{0}=\frac{p k}{\delta \gamma \varepsilon}>1
$$

$R_{0}$ is known as the basic reproductive number, representing a threshold condition for infection success occurring when $R_{0}>1$. $R_{0}$ thus defines the critical value for a second-order transition, as shown in Fig. 4 by tuning the infection rate $k$.

This only one among many different models of virushost interactions and in particular the role played by immune responses. Several monographs have explored diverse scenarios (Nowak and May 2000; Wodarz 2007;
Bocharov et al. 2018; see also Perelson and Weisbuch 1997 for a more physics-oriented view). Despite the potential richness and high-dimensional character of extended models, it is often feasible to find the conditions for successful propagation by means of simple models. This has also important consequences for defining the critical conditions for successful therapies (Perelson 2002).

\section{Epidemic thresholds}

Epidemiological models are diverse in their structure and complexity, depending on how many categories of individuals are included and whether they are deterministic or incorporate stochastic components. The simplest possible model is the susceptible-infected-susceptible (SIS) one, that only includes susceptible $(S)$ and infected $(I)$ individuals. This is a toy model, and some basic assumptions are required. The first one being that $I+S=N$, which means that, at some scale, the total population remains constant. Secondly, there is no heterogeneity and thus all interactions between individuals are weighted with exactly the same parameter values. In a well-mixed system, the rules outlined above can be described with two reactions associated to infection and recovery, given by:

$$
\begin{array}{r}
I+S \stackrel{\mu}{\longrightarrow} 2 I, \\
I \stackrel{\alpha}{\longrightarrow} S .
\end{array}
$$

It is easy to show that the equations describing our system are:

$$
\frac{d I}{d t}=\mu I S-\alpha I=-\frac{d S}{d t}
$$

and since the total population $I+S$ is conserved, using a normalized density of infected individuals $\rho=I / N$, we have

$$
\frac{d \rho}{d t}=\mu \rho(1-\rho)-\alpha \rho
$$

consistently with the previous result. We now have a one-variable model that can be solved analytically. This equation is actually the well-known logistic model with density-independent decay rate. This simple model is known to suffer a smooth transition as $\mu$ decreases below a given threshold (Fig. 5). Indeed, the mathematical picture of this smooth continuous phase transition is given by the so called transcritical bifurcation (Strogatz 1994).

Equation (22) can actually be re-written in a logisticlike form by defining $\rho^{*}=1-\alpha / \mu$, i.e.

$$
\frac{d \rho}{d t}=\mu \rho\left(\rho^{*}-\rho\right)
$$

If $\rho_{0}$ that represents the initial condition, the solution of this differential equation is:

$$
\rho(t)=\frac{\rho^{*}}{1+\left(\frac{\rho^{*}}{\rho_{0}}-1\right) e^{-(\mu-\alpha) t}} .
$$


After long periods of time, i.e., in the limit $\rho_{\infty}=$ $\lim _{t \rightarrow \infty} \rho(t)$ two solutions are possible, namely $\rho_{\infty}=\rho^{*}$ when $\mu>\alpha$ and $\rho_{\infty}=0$ when $\mu<\alpha$. The first point involves a stable epidemic event that would infect a fraction $(1-\alpha / \mu)$ of individuals of the population, whereas the second represents the extinction of the virus. The critical point $\mu_{c}=\alpha$ separates the subcritical phase, where the epidemics dies out from the supercritical phase, where the epidemics is self-maintained.

An important result from the previous model definition is that, at the supercritical phase, the initial growth of the epidemics is exponential. This can be shown by assuming that the current relative frequency of infected individuals is very small, i.e., $\rho \ll 1$. In this case, it is possible to make the approximation $1-\rho \approx 1$ and thus the equation for epidemic spreading now becomes:

$$
\frac{d \rho}{d t} \approx \alpha\left(R_{0}-1\right) \rho
$$

where we define $R_{0}=\mu / \alpha$ as the basic reproductive number. Using this definition, an exponential growth is obtained

$$
\rho(t)=\rho(0) e^{\alpha\left(R_{0}-1\right) t} .
$$

which is positive (and epidemic spreading occurs) provided that $R_{0}>1$ and the epidemic would die out if $R_{0}<1$. $R_{0}$ can also be seen as a the critical point at which the system transitions from no epidemic to epidemic success. Values of $R_{0}$ are quite variable among viruses. For example, it was estimated in the range 1.5 2.5 for the Ebola virus. It ranges from 2 to 5 for the sexual transmission of HIV-1, while the measles virus, which is airborne transmitted, has $R_{0}$ values ranging from 12 to 18 . It is interesting to note that $R_{0}$ involves several components, including the infectivity of the pathogen $\mu$, the recovery rate $\alpha$ and the population size $N$. Therefore, the behaviour of the critical point can be explored as a function of any of these three parameters, e.g., $\mu$ as shown in Fig. 5.

This very basic SIS model can be improved by adding specific spatial structure: hosts live in a lattice and the probabilities of transmission depend on the physical distances between individuals in the lattice. Obviously, this system makes computations slightly more complicated, but the conclusion is that a critical $R_{0}$ can still be recovered as a function of the rate arrival of infected individuals into the population, $\beta$, and the rate of recovery, $\alpha$ (Anderson and May 1998): $R_{0}=\beta / \alpha$, which still defines a second-order phase transition. The exact location of the transition point has moved and the shape of the curve on the right hand side (Fig. 5) is slightly different, but the phenomenon itself remains preserved.

\section{Large-scale pandemics on networks}

In the previous section we have considered in certain detail the very simple SIS model of epidemic spreading

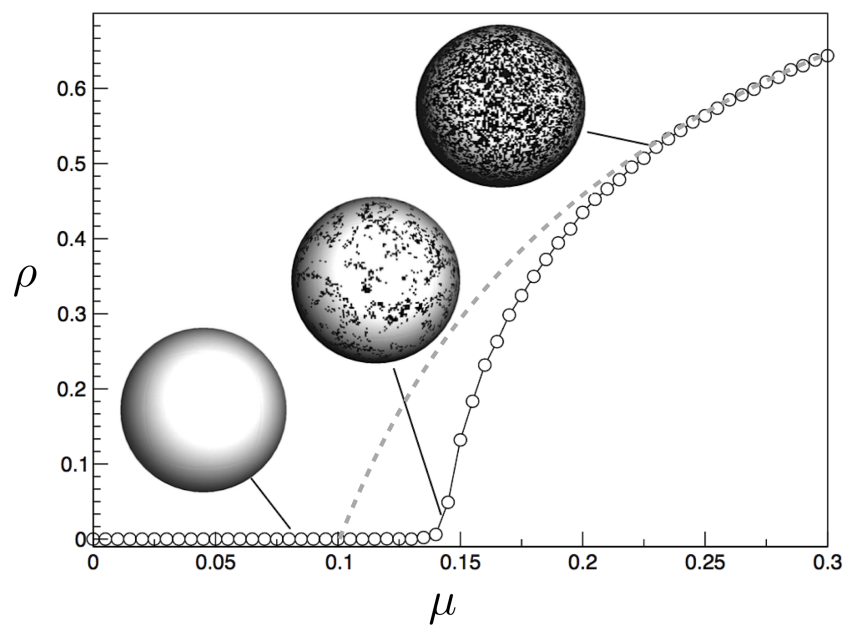

FIG. 5: Epidemic spreading as a continuous transition (dashed line). Here we use $\alpha=0.1$ and plot the stationary fraction of infected individuals $(\rho)$ against the infection rate, $\mu$. For comparison, we also display the same plot for a spatially extended system on a two-dimensional $100 \times 100$ square lattice. Here, infected and susceptible sites are shown as black and white patches. The spatial effects make more difficult the propagation of the epidemics and thus the critical point predicted by the mean field theory is found at higher values of $\mu$.

that occurs in a well mixed (mean field) context. Similar results would be found by assuming that individuals interact at random with a given probability. However, the networks of interactions among humans can depart from the mean field approach. Similarly, transportation networks connecting humans on large scales strongly depart from these simplified pictures (Pastor-Satorras and Vespignani 2001; Lloyd and May 2001; Brockman and Helbing 2013; Pastor-Satorras et al. 2015; Barabási 2016).

If we look at the probability $P(k)$ of a node $(e . g$. , an airport) being connected to $k$ other nodes, they follow heavy-tailed distributions described as scaling laws, i.e. $P(k) \sim k^{-\gamma}$, where $\gamma$ is known as the scaling exponent. Here we will explore the problem of epidemic spreading in scale-free networks by means of a SIS model. Each node in the graph of interactions represents and individual and each link a potential transmission event. The average density of infected individuals $\rho(t)$ (prevalence) at the mean-field level is

$$
\frac{d \rho(t)}{d t}=\mu\langle k\rangle \rho(t)[1-\rho(t)]-\alpha \rho(t),
$$

where $\mu$ is the infectivity of the virus and $\langle k\rangle$ the average degree of the graph (i.e. the number of connections per node). As we can see, we have just recovered the mean field equation for the SIS model, given by Eq. (22).

By defining an effective spreading rate $\lambda=\mu / \alpha$, we can write:

$$
\frac{d \rho(t)}{d t}=\lambda\langle k\rangle \rho(t)[1-\rho(t)]-\rho(t) .
$$


a

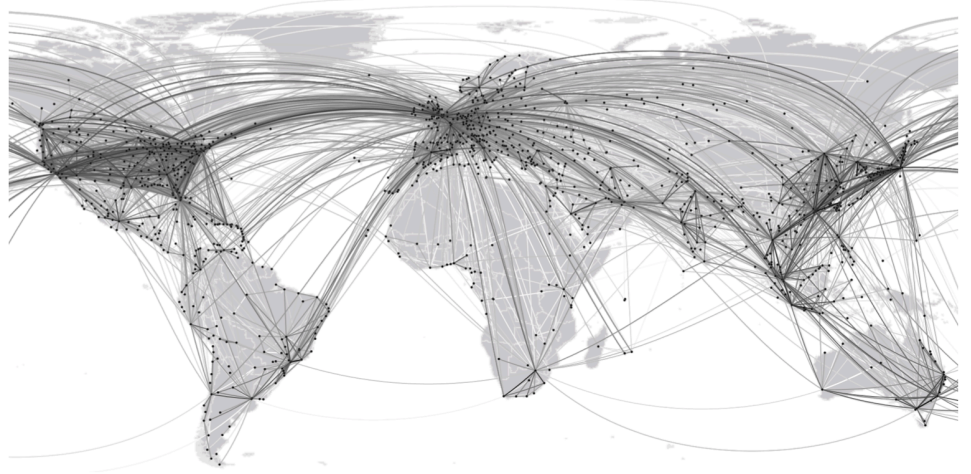

$\mathrm{b}$

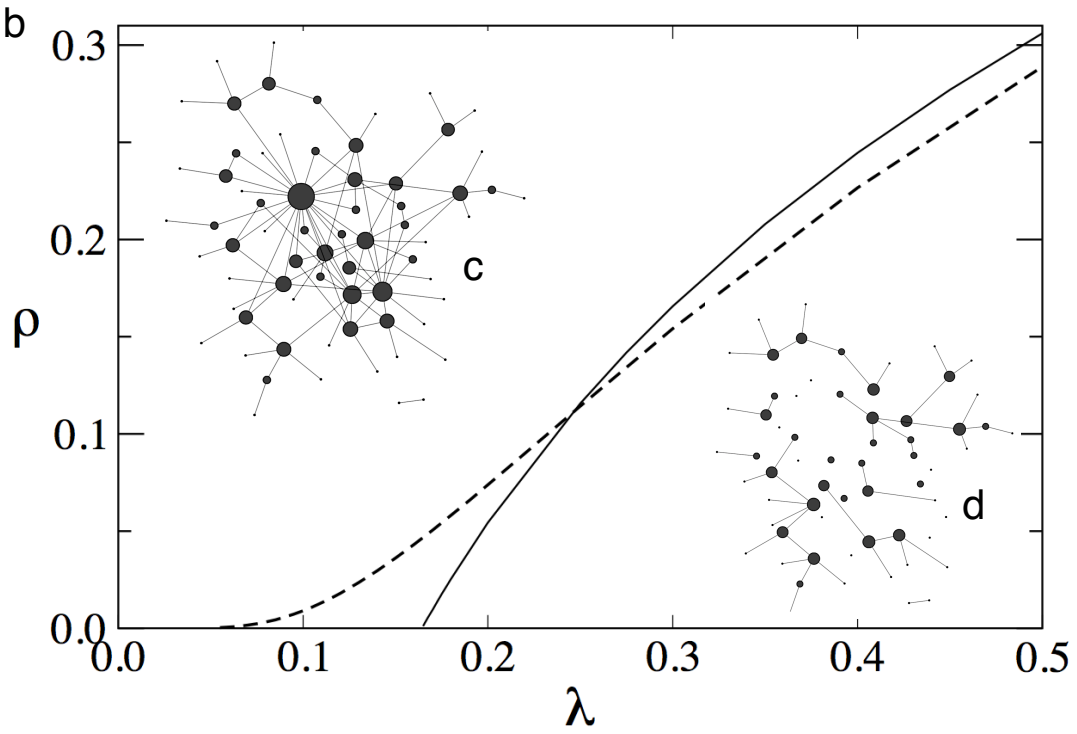

FIG. 6: Lack of eradication thresholds in epidemic models on scale-free networks. Large-scale transportation networks, such as the international web of airports (a) display highly heterogeneous connectivity distributions (picture after Dirk Brokman). (b) Mean field model prediction displaying a phase transition (in a SIS mode, continuous line) is compared with the one shown for a scale-free network (dashed line). When dealing with disease transmission networks among humans, which also display scale-free structure (c), protecting the hubs can effectively make the web homogeneous (d) thus recovering the standard, second-order transition found in homogeneous graphs(d). Insets c-d adapted from Barabási (2016).

The benefit of this mean-field equation stems from the fact that density correlations are ignored. On random graphs and related graphs, one can assume that $k \simeq\langle k\rangle$. It is easy to demonstrate that a non-zero epidemic threshold exists at $\lambda_{c}=\langle k\rangle^{-1}$ such that

$$
\begin{array}{ll}
\rho=0 & \text { if } \lambda<\lambda_{c}, \\
\rho \sim 1-\frac{1}{\langle k\rangle} & \text { if } \lambda \geq \lambda_{c} .
\end{array}
$$

So far, everything seems pretty much the same, but a crucial property of scale-free networks changes everything (Pastor-Satorras and Vespignani 2001; Lloyd and May 2001; Dezsö and Barabási 2001; Barabási 2016). The fluctuations $\left\langle k^{2}\right\rangle$ in scale-free networks diverge for any value of the critical exponent $2<\gamma<3$ and thus highly connected nodes are statistically significant: the mean field approximation breaks down. In order to take into account these fluctuations, the relative density $\rho_{k}(t)$ of infected nodes with given connectivity $k$ must be taken into account. The mean-field equations can thus be written as

$$
\frac{d \rho_{k}(t)}{d t}=\lambda k\left[1-\rho_{k}(t)\right] \Theta(\rho(t))-\rho_{k}(t)
$$

for $k=1, \ldots N$. A new term $\Theta(\rho(t))$ indicates the probability that any given link points to an infected node. The probability that a link points to a node with $k$ links is proportional to $k P(k)$. A randomly chosen link is thus more likely to be connected to an infected node with high connectivity, yielding

$$
\Theta(\rho(t))=\frac{\sum_{k} k P(k) \rho_{k}(t)}{\sum_{k} k P(k)},
$$

where $\sum_{k} k P(k)=\langle k\rangle$ by definition. At the steady state 
$d \rho_{k}(t) / d t=0$, and hence

$$
\rho_{k}=\frac{\lambda k \Theta}{1+\lambda k \Theta}
$$

and the following relation follows:

$$
\Theta=\frac{1}{\langle k\rangle} \sum_{k} k P(k) \frac{\lambda k \Theta}{1+\lambda k \Theta},
$$

where $\Theta$ is now a function of $\lambda$ alone.

The solution $\Theta=0$ is always satisfying the previous equation. A non-zero stationary prevalence $\left(\rho_{k} \neq 0\right)$ is obtained when the right hand and the left hand sides of Eq. (34), expressed as function of $\Theta$, cross in the interval $0<\Theta \leq 1$ allowing a nontrivial solution. It is easy to realize that this corresponds to the inequality

$$
\frac{d}{d \Theta}\left(\frac{1}{\langle k\rangle} \sum_{k} k P(k) \frac{\lambda k \Theta}{1+\lambda k \Theta}\right)_{\Theta=0} \geq 1
$$

being satisfied, defining the critical epidemic threshold by:

$$
\frac{\sum_{k} k P(k) \lambda_{c} k}{\langle k\rangle}=\frac{\left\langle k^{2}\right\rangle}{\langle k\rangle} \lambda_{c}=1,
$$

or rewritten as

$$
\lambda_{c}=\frac{\langle k\rangle}{\left\langle k^{2}\right\rangle}
$$

which is nothing but the inverse of the coefficient of variation of the network's degree (CV, Anderson and May 1991). This result means that in scale-free networks with $\gamma \in(2,3) \lambda_{c}=0$ (see dashed line in Fig. 6b) and thus for any value of $\lambda$ the infection can pervade the system with a finite prevalence (Pastor-Satorras and Vespignani 2001). For small $\lambda$ it is possible to solve explicitly the previous equation and calculate the prevalence in the endemic state as follows:

$$
\rho=\sum_{k} P(k) \rho_{k}
$$

In the particular case of the Barabási-Albert network with $\gamma=3$, we have $\rho \sim \exp (-C / \lambda)$ where $C$ is a constant.

The absence of any epidemic threshold in this network can be understood by noticing that in heterogeneous systems $R_{0}$ contains a correction term linearly dependent on the standard deviation of the connectivity distribution. In scale-free networks the divergence of the connectivity fluctuations always leads to an $R_{0}>1$ at any rate $\lambda$. This ensures that epidemics always have a finite probability to survive indefinitely. This of course makes more difficult to properly exploit a propagation threshold and define containment strategies. However, it is possible to "cure" the hubs (by immunizing them) and generate an effective threshold (Dezso and Barabási 2002; see also Kitsak et al.
2010; Wang et al. 2016; see also Hufnagel et al. 2004). The key idea here is that, once these highly connected nodes are protected from infection, the remaining graph is not fat-tailed anymore, as shown by the inset graph in Fig. 6d. The presence or absence of epidemic thresholds in the real world has thus considerable implications, both in the context of disease spreading and the propagation of computer viruses (see Solé and Elena 2019 and references therein).

\section{THE EVOLUTIONARY DIMENSION: MULTIPARTITE VIRUSES AND MUTUALISM}

The previous examples illustrate how simple dynamical models can display marked changes in the behavior of a diverse range of problems in virology. Except for the self-assembly problem, all these case studies share a unifying feature: in a way or another, the transition requires achieving a critical threshold level of infection beyond which propagation occurs. There is, however, another layer of complexity beyond these two-phase systems that has to do with the nature itself of ecological interactions and their impact on virus evolution. Crossing thresholds in these cases has consequences for the presence or absence of cooperative interactions among different viral components.

Transitions between different kinds of population level dynamics, such as competition versus mutualism or parasitism, are particularly important for evolutionary biology. What kind of transitions can be found in models of virus dynamics? A very interesting example is given by a particular class of RNA viruses known as multipartite viruses (Iranzo and Manrubia 2012; Sicard et al. 2016, 2019; Lucía-Sanz et al. 2018). In a multipartite virus, no single viral particle contains all the genomic segments, which appears segregated in two or more particles. As a consequence, the information required for a full infection cycle is not linked into a single molecule but distributed.

An example of multipartite virus (Fig. 7a) is tobacco rattle virus (TRV), which infects, along with tobacco, many other plant species. It consists of two particles, a long and a short one. The first contains the $R N A_{1}$ segment encoding for the replicase, while the second particles encapsulates $R N A_{2}$ segment that encodes for the coat protein. As a consequence, the long particle can infect and trigger the disease on its own, but the $R N A_{1}$ will be trapped in the plant unless the coat protein is present. A full cycle of infection thus requires the two particles to be present. This kind of constraint creates somewhat of a puzzle: viruses are typically seen as very efficient, optimal replicators, but the picture of viruses split into different particles seem to contradict this picture. What are the consequences of this genome splitting? A model brought forward by Nee (2000) illustrates the richness of population models of multipartite viruses and the presence of different types of ecological interactions. The model includes three variables and the tran- 
a

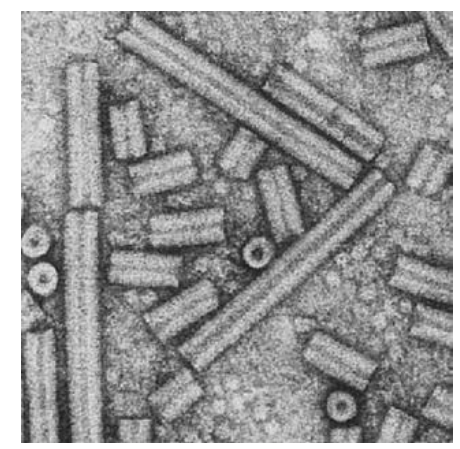

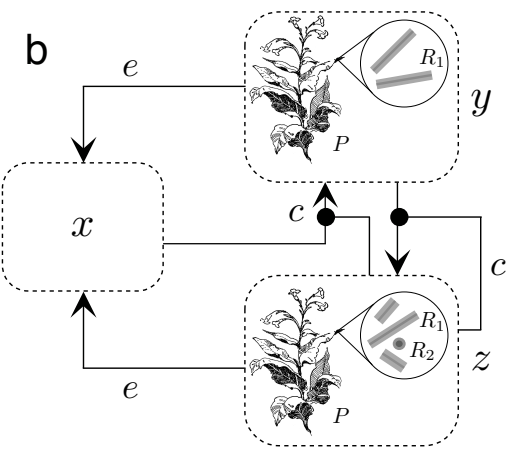

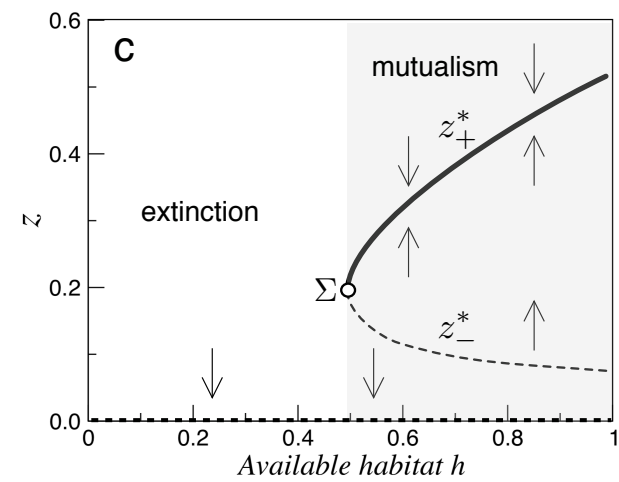

FIG. 7: Multipartite viruses include two or more genomic segments, encapsidated independently, and all required to complete the full life cycle of the virus. An example is tobacco rattle virus TRV, shown in (a) where we can appreciate the presence of two kinds of particles (image by John Antoniw). A simple metapopulation model with a simple set of transition rules (b) can help to explore the population and evolutionary traits of multipartite viruses. In a simple model of multipartite virus dynamics (Nee 2000) the previous set of rules led to a sharp transition phenomenon (c) where the abundance of plants carrying the two components of the multipartite virus are indicated against $h$.

sitions between states, as described in Fig. 7b. Here a given habitat is considered, which can be visualized as a grid of patches, as usual in many models of metapopulation dynamics (Mollanen and Hanski 1998; Hanski 1999). Patches can be empty, occupied by a plant carrying only $R N A_{1}$ or plants carrying the two components of the multipartite virus. These populations (normalized) are indicated as $x, y, z$ respectively. A fraction $0 \leq h \leq 1$ of this habitat is occupied.

Two main events are considered here: colonization, at a given rate $c$ and extinction, at a rate $e$. Both are probabilities and thus $0 \leq c, e \leq 1$. The basic model defining the dynamics of each component is given by (Nee 2000):

$$
\begin{aligned}
& \frac{d x}{d t}=e y+e z-c z x \\
& \frac{d y}{d t}=-e y+c z x-c z y \\
& \frac{d z}{d t}=-e z+c z y,
\end{aligned}
$$

with a normalization condition defined by:

$$
h=x+y+z \text {. }
$$

This constraint allows to reduce our three-equation model to a two-dimensional system, $i$. e.

$$
\begin{aligned}
& \frac{d y}{d t}=-e y+c z(h-y-z)-c z y, \\
& \frac{d z}{d t}=-e z+c z y .
\end{aligned}
$$

The equilibrium points obtained from $d y / d t=d z / d t=$ 0 , are (after some simple algebra)

$$
\begin{aligned}
& x^{*}=h-y^{*}-z^{*} \\
& y^{*}=\frac{e}{c} \\
& z_{ \pm}^{*}=\frac{1}{2}\left(h-\frac{2 e}{c} \pm \sqrt{h^{2}-\frac{4 e h}{c}}\right) .
\end{aligned}
$$

A domain involving three possible equilibrium points (two of them stable) is given by the condition

$$
h^{2}-\frac{4 e h}{c} \geq 0
$$

i. e. the argument within the square root needs to be positive. This will occur provided that

$$
h>h_{c}=\frac{4 e}{c} .
$$

This is illustrated in the bifurcation diagram in Fig. $7 \mathrm{c}$, where the possible equilibrium values of $z$ are plotted against the available population size $h$. The discontinuous jump separating the phases of mutualism and extinction is given by the so-called saddle-node bifurcation, which often arises in systems with positive feedbacks such as facilitating or cooperation. The diagram thus indicates that a minimal accessible number of individuals (plants in our metapopulation context) is required to allow for the propagation of the multipartite viruses. This is indicated by the extinction phase (left), separated by a mutualistic, persistence phase (gray domain). The positive solution starts at a value $z^{*}=\Sigma$ defining the minimal (critical) population size. This result is related to those found within the context of extinction thresholds in metapopulation models due to habitat loss and fragmentation including continuous (Bascompte and Solé 1996) and first-order (Sardanyés et al. 2019) transitions. It thus connects the problem of reduced habitat and the persistence of viral associations requiring higher-order interactions (such as mutualism).

Beyond this simplistic model, other model approaches to multipartite viruses incorporate other types of transitions, such as transitions from monopartite to multipartite life cycles (Valdano et al 2019). These models are an essential part towards an understanding of the evolutionary origins of multipartite viruses and how they 
are affected (among other things) by the multiplicity of the infection (Lucia-Sanz and Manrubia 2017). The full analysis of these models reveal a rich phase space where diverse phases include competition, mutualism and commensalism.

\section{INFORMATION AND DIVERSITY TRANSITIONS}

In this section we expand our previous case studies of phase transitions with three more examples. These cases are not related to transitions at different levels of virus organisation, as we have discussed so far, but with another type of phenomena: phase transitions involving changes in information and genetic diversity. The first example is the well known phenomenon of the meltdown of genetic information at increasing mutation rates, with viral populations entering into the so-called error catastrophe regime. This is a nice example of second-order phase transition. The second example has to do with the dual role of homologous recombination in the rescue of mutationally compromised populations if recombination is at some critical value, or driving them to extinction if recombination is far too high. Interestingly, the first situation corresponds to a second-order phase transition, while the second situation corresponds to a first-order one. The third example deals with the role of withinhost HIV-1 diversity in the progression towards AIDS, which is also a second-order phase transition.

\section{A. The error catastrophe}

Because of their intrinsic simplicity and their strong dependence upon the host molecular machinery to complete their cellular infection cycle, viruses are unique dynamical systems. One particularly important trait of RNA viruses is their high mutation rates, much higher than any other rates exhibited by cellular systems and a consequence of the lack of repair mechanisms associated to their RNA-dependent RNA polymerases (the inset in Fig. 8 shows the structure of hepatitis $\mathrm{C}$ virus replicase). This enzyme catalyses the replication of RNA templates producing new RNAs and mutation rates per nucleotide and replication cycle are in the range $10^{-4}-10^{-5}$ (Sanjuán et al. 2010). Recent research has also determined mutation rates of about $10^{-3}$ per base per cell in the revers transcriptase of HIV-1 (an RNA-dependent DNA polymerase) in peripheral blood mononuclear cells (Cuevas et al. 2015). In DNA-based systems, such as the cellular hosts, the process of DNA polymerization is usually associated to a proofreading and highly efficient and redundant repair mechanisms that effectively reduces mutation rates to a range $10^{-8}-10^{-11}$ ensuring a controlled replication cycle (Drake et al. 1998). Since high mutation carries a burden of genetic errors, this implies

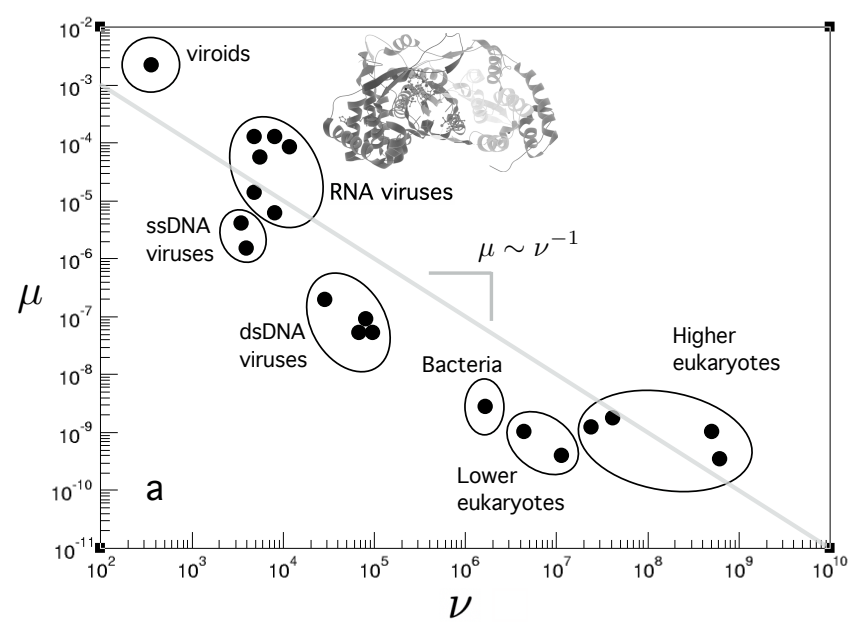

FIG. 8: Scaling law in the per-site mutation rate $(\mu)$ versus genome size $(\nu)$, shown in log-log scale. The plot includes chosen examples of RNA viruses, which lack error-repairing mechanisms during replication (the inset displays the structure of hepatitis $\mathrm{C}$ virus RNA replicase (image obtained from the wikipedia)). Larger genomes are represented by both single-stranded and double-stranded RNA viruses and DNA viruses, bacteria and a few eukaryotes (adapted from Gago et al. 2009). The continuous line is used to highlight the inverse law linking mutation rate $\mu$ and genome length $\nu$ predicted by the error threshold theory.

that many resulting viral genomes can contain deleterious changes leading to non-viable viral particles.

Mutation is a crucial component of evolution, as genetic variability is the fuel on which natural selection operates to adapt populations to their environment. In this sense, an error-prone polymerase can be seen as useful to keep pace with the always changing environmental conditions in which RNA viruses live (Domingo 2000). However, keeping in mind that mutation is a random process independent on the value that mutations may have in the future generations, mutation itself is a double-edge sword: too many mutations per genome may simply drive fitness levels to such a low values that would not be compatible anymore with a successful replication. Therefore, mutation rates, as any other trait, have evolved and have been optimized for the lifestyle of RNA viruses: just high enough but not more (Elena and Sanjuán 2005). For RNA viruses, a heterogeneous population results in a socalled viral quasispecies (Eigen 1971; Eigen et al. 1987; Schuster 1994; Domingo and Holland 1994; Domingo et al. 1995; Domingo et al. 2012). A viral mutant swarm can be seen as a group of genomes dominated by a master sequence of high fitness that may, or may not, coincide with the average sequence of the population, the consensus sequence.

The quasispecies population structure has many implications for the biology of RNA viruses. The most important is that the mutant swarm stores a reservoir of phenotypes crucial to cope with environmental uncertainties: 

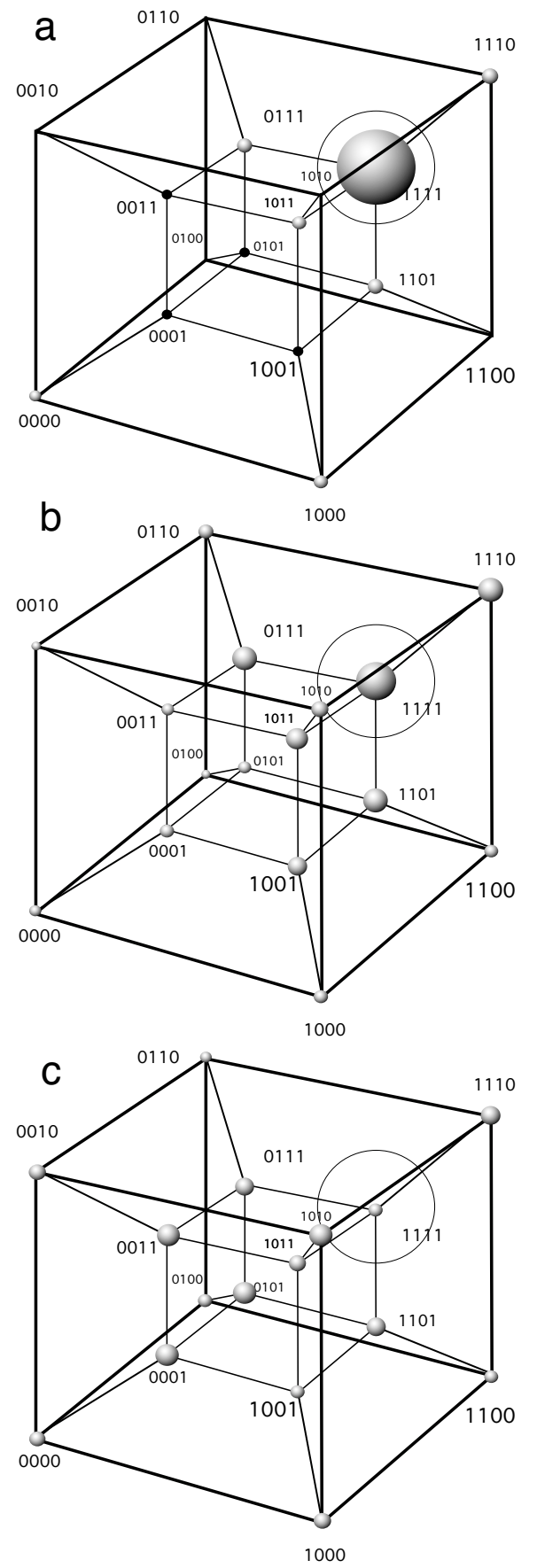

FIG. 9: The error catastrophe on a four-dimensional hypercube. Three occupation patterns for 4-bit strings are shown: strings 1111 have a large fitness $f_{m}$, whereas all $s_{1} s_{2} s_{3} s_{4} \neq 1111\left(s_{i} \in\{0,1\}\right)$ have the same, smaller fitness $f$. Strings replicate with a mutation rate $\mu$ per bit. (a) Starting with all 1111 strings, very small $\mu$ leads to a large majority of strings located at 1111. (b) Larger mutation generates a more disperse mutant cloud still surrounding the node 1111 (b). (c) A mutation rate close to its critical value allows to preserve a cloud of strings while generating much more diversity and keeping the information of sequence 1111. Beyond the threshold, the population experiences pure genetic drift and the master sequence is not found at all. within the context of the virus infection and pathogenesis, that includes the host responses tied to immunity but also others such as tissue specificity or resistance to drugs (Andino and Domingo 2015; Domingo et al. 2012; Lauring and Andino 2010; Holmes 2010).

One particularly unexpected consequence of the quasispecies nature of viral populations is deeply connected to the informational nature of RNA viruses. This is known as the error catastrophe problem (Eigen 1971; Eigen et al. 1987; Schuster 1994; Domingo and Holland 1994) and is tightly related with second-order phase transitions. It was originally defined within the context of an abstract population of mutating molecular replicators competing for limited resources. More precisely, Eigen and Schuster considered a large population of genomes where each sequence could replicate at some fix rate. Replication rate will be sequence-dependent and the relation between sequence and growth rate should be expected to be complex. Additionally, it is assumed that every time a string replicates, mutations can occur at a given rate $\mu$.

Eigen (1971) predicted that there is a critical mutation rate, $\mu_{c}$, that decays as $\mu_{c} \sim 1 / \nu$ beyond which no Darwinian selection can occur, and thus no viable sequences would be observable for mutations higher than $\mu>\mu_{c}$. In that case, random drift would be observed. Instead, below the threshold, information can be maintained in stable ways. Experimental data confirms this inverse relationship. and thus mutation rates decrease as an inverse power law of genome length (Fig. 8). RNA viruses exhibit the highest rates, orders of magnitude larger than DNA viruses. At this point, two questions emerge: what is the origin of such relationship? And what are the limits (if any) of mutation rates in RNA viruses?

Eigen-Schuster quasispecies model (Eigen 1971) considers a set of populations $\left\{x_{i}\right\}$ representing the abundance of different genomes, changing in time by the following set of dynamical equations:

$$
\frac{d x_{i}}{d t}=\sum_{j=1}^{n} f_{j} \mu(j \rightarrow i) x_{j}-\Phi(\mathbf{x}, t) x_{i}
$$

where $x_{i}$ indicates the fraction of the population associated to the $i$-th mutant genome equipped with a $M$ letter alphabet (here $i=1, \ldots n$, where $n=M^{\nu}$ is very large, $\nu$ being the length of the genome) so that a normalization condition applies, namely: $\sum_{j=1}^{n} x_{j}=1$. Here $f_{j}$ is the growth rate of the $j$-th mutant, $\mu(j \rightarrow i)$ is the probability of having a mutation from sequence $j$ to sequence $i$ and $\Phi(\mathbf{x})^{c}$ is the average fitness associated to

\footnotetext{
${ }^{c} \Phi(\mathbf{x})$ is usually termed as the dilution outflow, which ensures a constant population $\left(\sum_{i=1}^{n} x_{i}=\right.$ constant and $\left.\sum_{i=1}^{n} \dot{x}_{i}=0\right)$ also introducing competition between replicating genomes
} 
the population vector $\mathbf{x}=\left(x_{1}, \ldots x_{n}\right)$, i.e.,

$$
\Phi(\mathbf{x}, t)=\sum_{j=1}^{n} f_{j} x_{j}\left(\sum_{i=1}^{n} \mu(j \rightarrow i)\right)=\sum_{j=1}^{n} f_{j} x_{j}=\langle f\rangle .
$$

This model can sometimes be treated analytically under a number of well defined set of conditions, showing that the population structure corresponds to a cloud of sequences (Eigen 1971; Eigen et al. 1988, 1989; Schuster 1994).

In this section we consider a specific case that will illustrate how mutation can sharply limit the length of genomes and thus the amount of information stored in a quasispecies. Many possible extensions are not considered here, such as the introduction of spatial degrees of freedom (Altmeyer et al 2001; Pastor-Satorras and Solé 2001; Aguirre and Manrubia 2008; Sardanyes and Elena 2011), secondary RNA structure (Stitch et al. 2007) or more complex fitness landscapes (Saakian et al 2006; Saakian 2018).

The problem considered here can be mapped into a high-dimensional sequence hyper-cube (Fig. 9), where each string is a digital genome, connected to nearest neighbors in the cube that differ by one bit (single mutation), considering binary genomes i.e. $M=2$. Without loss of generality, the general model described above can be collapsed into only two fitness classes, namely $f_{m}$ for the master and $f$ for any other sequence of the mutant spectrum (i.e., $f_{1}=f_{2}=\ldots=f_{n}=f$ ) where $n$ is very large $(n \gg 1)$. Hereafter it is assumed that $f_{m}>f$, i.e., the master sequence replicates more efficiently than any other sequence (Swetina and Schuster 1982). Assuming that i.e., $\mu(i \rightarrow j)=\mu$, we can split our system of equations into two sets: the master sequence and the mutant sequences. The system presented below is only a simplified approximation to the space connecting different genomes. A more accurate picture is provided by Fig. 9(b): from a given population $x_{j}$, mutation will not lead back to the master sequence or will be difficult to occur. In this case, we will have $x_{m}+x=1$ where we use $x=\sum_{j} x_{j}$. For the master sequence we get:

$$
\frac{d x_{m}}{d t}=f_{m}(1-\mu) x_{m}+\sum_{j=1}^{n} f_{j} \frac{\mu}{n} x_{j}-x_{m} \Phi(t),
$$

whereas the set of equations for the mutant sequences reads:

$$
\frac{d x_{i}}{d t}=\frac{f_{m} \mu}{n} x_{m}+\sum_{j=1}^{n} f_{j} \frac{\mu}{n} x_{j}-x_{i} \Phi(t) .
$$

It is easy to show, after some algebra, that the first equation can be simplified to

$$
\frac{d x_{m}}{d t}=\approx f_{m}(1-\mu) x_{m}-x_{m} \Phi(t)
$$

where we have used $\mu / n \ll 1$. For the equation describ- ing the mutant class, we have:

$$
\frac{d x_{i}}{d t}=\frac{f_{m} \mu}{n} x_{m}+f(1-\mu) x_{i}+\sum_{j \neq i}^{n} \frac{f \mu}{n} x_{j}-x_{i} \Phi(t) .
$$

Since the ultimate goal is to describe the dynamics of all these populations together, so we made the sum over all possible mutant genomes, i.e.,

$$
\begin{aligned}
\sum_{i=1}^{n} \frac{d x_{i}}{d t} & =\sum_{i=1}^{n} \frac{f_{m} \mu}{n} x_{m}+\sum_{i=1}^{n} f(1-\mu) x_{i} \\
& +\sum_{i=1}^{n} \frac{f \mu}{n}\left(\sum_{j \neq i}^{n} x_{j}\right)-\sum_{i=1}^{n} x_{i} \Phi(t) \\
=\mu f_{m} x_{m}+ & f(1-\mu) x+f \mu\left(x-x_{i}\right)-x \Phi(t) .
\end{aligned}
$$

Given the homogeneous mutation rates, a stationary distribution will lead to $x_{i} \approx x / n$ and thus the approximation $x-x_{i} \approx x$ is guaranteed, leading to

$$
\frac{d x}{d t} \approx \mu f_{m} x_{m}+f x-x \Phi(t)
$$

and as a result of these approximations, the twocompartment model (Fig. 9(a)) can be collapsed into a one-dimensional system (Swetina and Schuster 1982):

$$
\begin{aligned}
\frac{d x_{m}}{d t} & =f_{m}(1-\mu) x_{m}-x_{m} \Phi\left(x_{m}, x\right), \\
\frac{d x}{d t} & =f_{m} \mu x_{m}+f x-x \Phi\left(x_{m}, x\right) .
\end{aligned}
$$

As we did above for the replicator model, the condition $x_{m}+x=1$ leads to $\Phi(t)=\langle f\rangle=f_{m} x_{m}+f x$. Using this function, and since $x=1-x_{m}$, it can be obtained after some algebra an equation for the master sequence:

$$
\frac{d x_{m}}{d t}=x_{m}\left[\left(1-x_{m}\right)\left(f_{m}-f\right)-\mu f_{m}\right]
$$

Two alternative equilibria are possible. The first one being $x_{m}=0$, that corresponds to the extinction of the master sequence and the full dominance of the pool of mutants, and a nontrivial equilibrium

$$
x_{m}=1-\frac{\mu f_{m}}{f_{m}-f},
$$

allowing the coexistence of both master and mutant sequences. The later solution will be positive (and the master sequence will be present) provided that $x_{m}>0$ and this will occur if the mutation rate is lower than the critical value:

$$
\mu<\mu_{c}=1-f / f_{m}
$$

This expression defines the boundary separating two well defined phases, shown in Fig. 10(a). Within the quasispecies phase, master genomes will be present, along with a tail of mutants. Once the boundary is crossed, all 


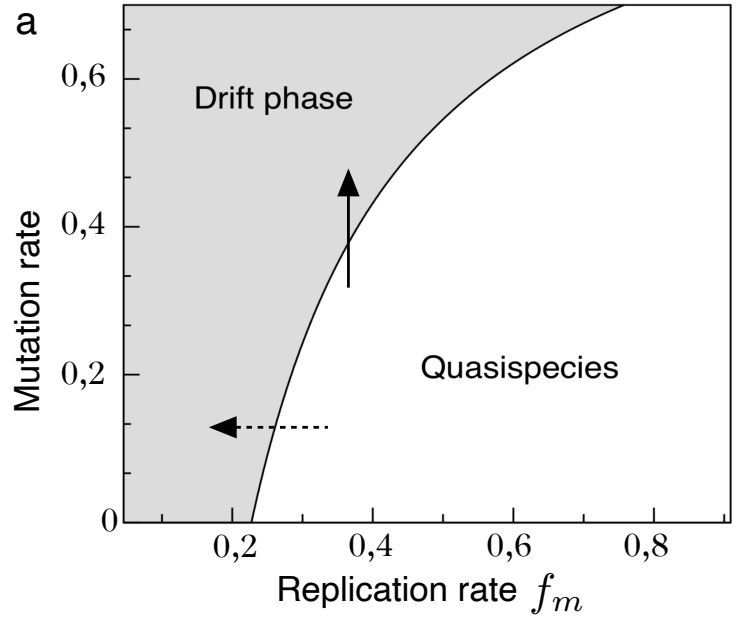

b
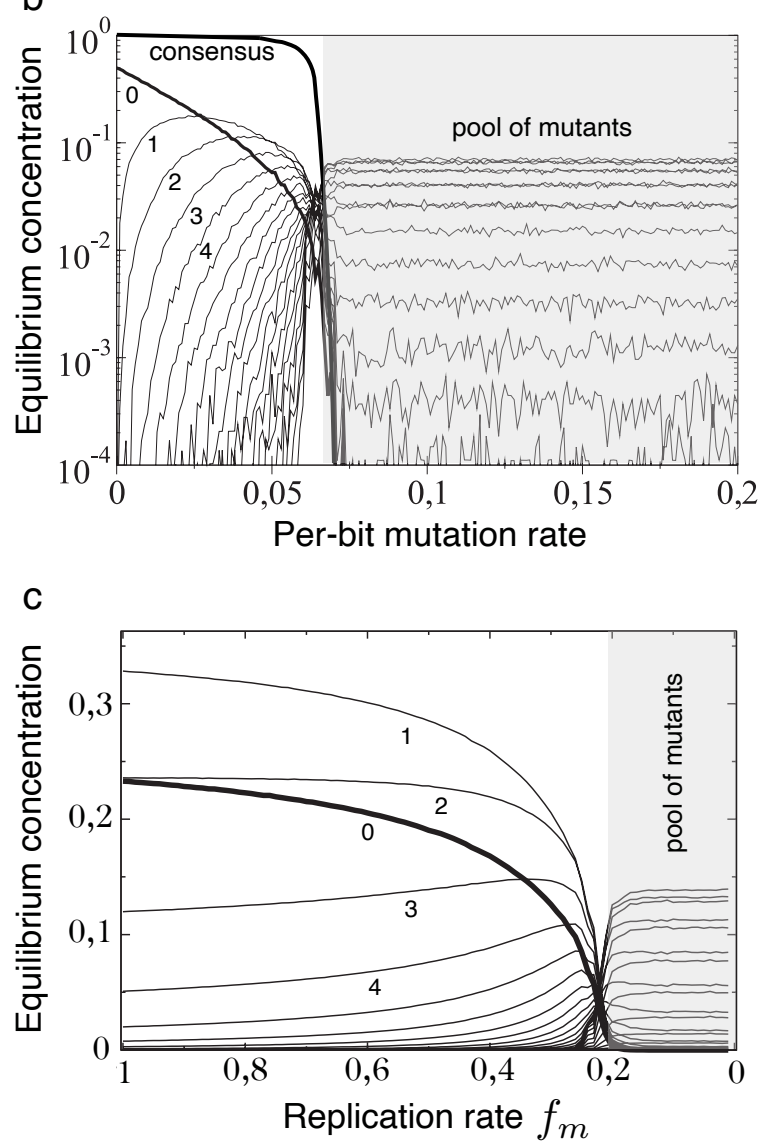

FIG. 10: The error catastrophe as a continuous phase transition: by plugging all non-master sequences into a single class, the mean field model (a) predicts two phases separated by a critical curve given by Eq. (62). The quasispecies distributions can be also studied with in silico bit-strings simulation models (the small numbers denote the sequences with 0 (master) and 1,2,3,4 mutations). Two examples are shown in panels (b) and (c). As Eq. (62) predicts, the error threshold can be achieved by either tuning mutation and replication rates. master sequences disappear, despite their higher replicating efficiency, and only mutant genotypes remain in the population (gray area in panels (a-c) in Fig. 10). This boundary actually corresponds to a transcritical bifurcation (Sardanyés 2009, see also Bull et al. 2005). The phase transition tied to the error threshold can be obtained with in silico evolutionary models (Sardanyés et al. 2009, Elena et al. 2010, Sardanyés and Elena 2011). Panels (b) and (c) in Fig. 10 display results using bit-string simulations, respectively (see the next paragraph for the meaning of mutation in bit-string systems). Specifically, they show how the stationary distribution of sequences change at increasing mutation rate (solid arrow in Fig. 10(a)) and decreasing the replication rate of the master sequence (dashed arrow in Fig. 10(a), see also Solé et al. (2006)).

As discussed above, a scaling law seems to connect the mutation rate of a given genome and its length (Fig. 8). We are now in the position of mathematically deriving this remarkable dependence. In the previous calculations, $\mu$ defines the mutation rate per genome, but the accurate definition of mutation is to express it as changes per nucleotide site (i.e., relative to the string of length $\nu$ ). If $\mu_{b}$ is the mutation rate per site (per-bit mutation), it is not difficult to see that it is related to $\mu$ by:

$$
\mu=1-\left(1-\mu_{b}\right)^{\nu}
$$

Here $p=\left(1-\mu_{b}\right)^{\nu}$ is the probability that none of the units are mutated, the difference $1-p$ is just the probability that some unit (and thus the genome) does mutate. Since $\mu_{b}$ is typically very small, we have: $\mu \approx 1-e^{-\mu_{b} \nu} \approx$ $1-\mu_{b} \nu$.

If we return to the previous critical condition for mutation rates and write it down as a function of $\mu_{b}$ it turns out

$$
\mu_{b}^{c}=\frac{\alpha}{\nu}
$$

of where $\alpha>0$ is a scaling factor. The last expression actually corresponds to the observed inverse decay of mutation rates as an inverse of their genome size, as shown in Fig. 8.

This work has inspired the suggestion that deep connections exist between the critical threshold in RNA viruses and other transition phenomena such as cancer relapse when genomic instability grows beyond a (still to be tested) critical threshold (Solé 2003; Solé and Deisboeck 2004; Solé 2012; Castillo et al. 2017). Recent mathematical and computational investigations on cancer phenotypic quasispecies (Sardanyés et al. 2017, 2018; Sardanyés and Alarcón 2018) have also confirmed this prediction of a critical threshold involving cancer cells extinction under genomic instability.

As a final point, it is worth mentioning that the similarity between the quasispecies as described by Eigen's theory and the physics of phase transitions goes beyond the analogy. It is actually possible to show that there is actually a mapping between this theory and the dynamics of the two-dimensional Ising model (Leuthäusser 
1986, 1987; Tarazona 1992). Specifically, it is possible to establish a parametrization connecting mutation rate with a temperature parameter and the existence of a universal theory that pervades both classes of models. These results have been successfully applied to the development of a statistical physics of quasispecies (Di Collobiano et al. 2003; Saakian et al. 2006; Park et al. 2007; Saakian 2018).

\section{B. The double-edge sword of recombination}

The quasispecies model presented above defines a minimal approach to a largely complex problem, where other phenomena can play an important role. An important phenomenon, pervasive among RNA viruses, and that contributes to generate genetic variability along with mutation is recombination. To explore whether recombination also generates informational phase transitions, let us consider, once again, our simplified model where a twospecies (master and non-master) are used, but now we also introduce the possibility of recombination between individual genomes. This process implies an additional form of diversity generation as genomes not only display mutations but also get scrambled in many ways through recombination. How does the existence of genome recombination affect the presence of error thresholds?

One approximation involves the same minimal model just described in the previous section. Consider the previous landscape and a new phenomenon that affects the generation of new sequences. Now two strings can experience cross-over thus generating, at a given rate $r$, new strings from the old ones. The likelihood that such event takes place grows with the amount of sequences. It can be shown (Boerlijst et al. 1996) that the two-dimensional model with a single peak with recombination now reads:

$$
\begin{aligned}
\frac{d x_{m}}{d t} & =f_{m}(1-\mu) x_{m}-r x x_{m} \psi\left(x_{m}\right)-x_{m} \Phi \\
\frac{d x}{d t} & =f_{m} \mu x_{m}+f x-r x x_{m} \psi\left(x_{m}\right)-x \Phi .
\end{aligned}
$$

The second term in the right-hand side of the first equation accounts for recombination by means of interactions between master and non-master strings taking place at rate $r$. The additional term $\psi\left(x_{m}\right)$ weights recombination in terms of the frequency $x_{m}$. The simplest choice here is to assume a linear function, namely

$$
\psi\left(x_{m}\right)=\left(1-x_{m}\right) .
$$

To see the logic of this choice, consider the following argument. For small values of $x_{m}$, most sequences will be non-master, and thus recombination probability $1-x_{m}$ will be high. This makes sense since a whole repertoire of strings can recombine. This is the case shown schematically in Fig. 10a, where a three-dimensional landscape considering the recombination of the sequences 111 (master) and 000 (non-master) is shown. The event is indicated by the open circle with letter $\psi$. a

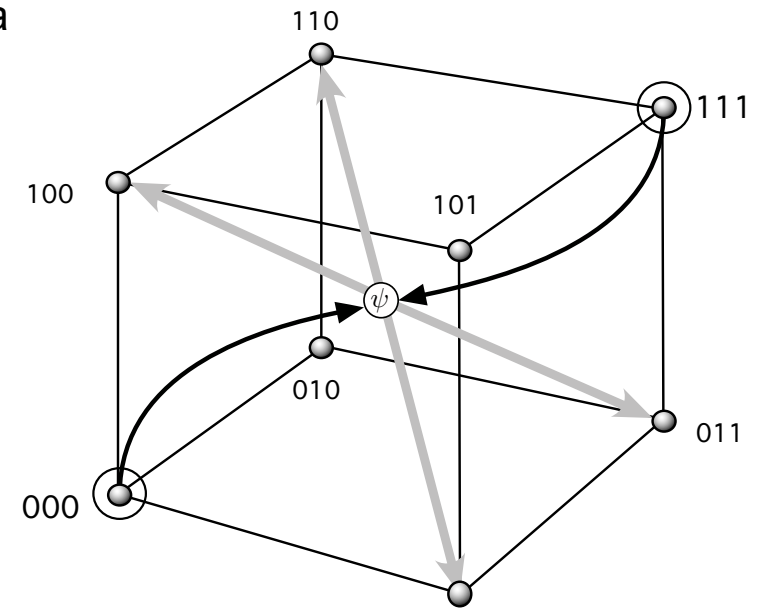

b

001

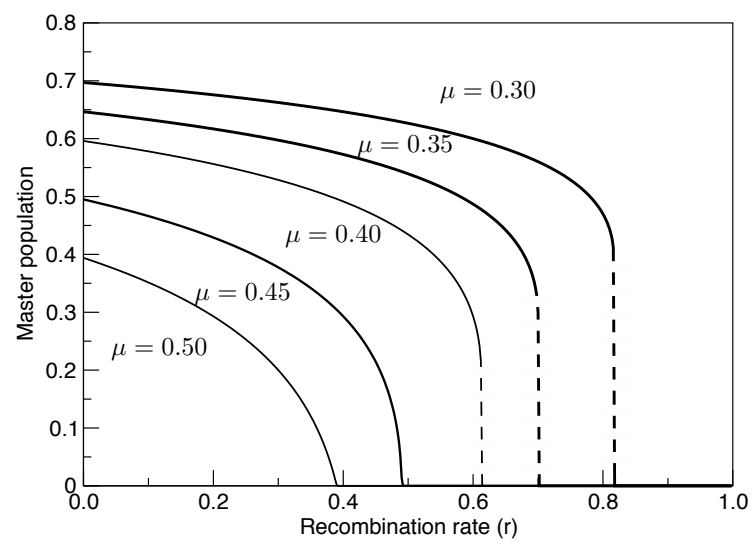

FIG. 11: Transitions in quasispecies with recombination. (a) Simple picture of a 3-dimensional sequence space. When the master sequence abundance is small, recombination between the master string 111 and another non-master one (here we use 000) gives new non-master strings. The two recombined sequences are highlighted with a circle and their interaction is indicated by the open circle with $\psi$ (the recombination function) at its center. The four possible outcomes of this event are indicated with grey arrows. (b) Transition diagrams obtained plotting $x_{m}^{*}$ against recombination rate with $f=10^{-2}$. Here the first order transitions are indicated with discontinuous lines.

A simple recombination event can generate several non-master strings (gray arrows). Instead, when the master sequence dominates (and $1-x_{m}$ will be small) recombination events will take place with the one-bit (single mutation) distance strings, essentially returning the same strings as a result.

Using our previous definitions, the equation describing the master sequence dynamics now reads:

$$
\frac{d x_{m}}{d t}=(1-\mu) x_{m}-r x_{m} x \psi\left(x_{m}\right)-\left[x_{m}+f\left(1-x_{m}\right)\right]
$$

where $x=1-x_{m}$ and, for simplicity, we have set $f_{m}=1$ (and thus $0<f<1$ ). With these choices, one obtains 
(after some simple algebra)

$$
\frac{d x_{m}}{d t}=x_{m}\left(1-x_{m}\right)\left[1-f-r\left(1-x_{m}\right)\right]-\mu x_{m} .
$$

These one-dimensional equation has a trivial equilibrium point $x_{m}^{*}=0$ and two potential extra equilibria $x_{m}^{*} \in$ $\left\{\Gamma_{-}, \Gamma_{+}\right\}$given by:

$$
\Gamma_{ \pm}=\frac{1}{2 r}\left[-(\phi-r) \pm\left((\phi+r)^{2}-4 r \mu\right)^{1 / 2}\right] .
$$

where we use $\phi=1-f-r$. In order to have real, positive solutions, it is not difficult to show that the following inequality for recombination rate is required:

$$
r \leq \frac{1}{4 \mu}(1-f)^{2}=r_{c}
$$

The critical value marks the domain where extinction is expected to occur (when $r=r_{c}$ ). However, the nature of the transition is different for smaller mutation rates, as summarized in Fig. 10b. For higher mutation rates, the decay of the master sequence involves a continuous phase transition. By contrast, for smaller mutation rates the dominant role of recombination changes the nature of the transition, that becomes of first-order. This is a typical outcome (a rather universal one) that is observed in a wide class of dynamical systems exhibiting cubic terms (Weissmann and Shnerb 2014; Fontich and Sardanyés 2008; Sardanyés et al. 2019). The introduction of a higher-order term that requires a density-dependent pairwise exchange is responsible for this phenomenon.

\section{Diversity thresholds in AIDS progression}

A final example of our list of viral transitions involves a theoretical model that was used to explain a paradoxical behavior displayed by the long-term progression of HIV-1 infected patients. An intriguing question that emerged in the early times of the pandemic was the presence of an asymptomatic period: before the symptoms associated with AIDS were detectable, measured viral load appeared very low. This was a paradox: if HIV-1 is responsible for the development of AIDS by efficiently killing their target $\mathrm{CD}^{+} \mathrm{T}$ cells, one should expect to see a growth in the number of viruses along with a constant suppression of $\mathrm{CD}^{+} \mathrm{T}$ cells until collapse is achieved. Instead, a very small virus load suggests that it is kept under control by the immune system. Perhaps AIDS had to do with other factors?

The solution for this conundrum was provided, to a large extent, by a mathematical model including the heterogenous nature of these populations and the arms race between the immune system. Such arms race creates the selection pressure required for the generation of HIV1 antigenic escape mutants. During the asymptomatic phase of infection, error-prone replication of HIV-1 generates increasing numbers of antigenic variants. The immune system would keep fighting with novel variants as it gets undermined by the interaction and more strains accumulate. The theory predicts that there is a threshold value in antigenic diversity above which the immune system cannot control the viral population, triggering immune system's collapse (Nowak et al. 1991; Nowak et al. 1996).

To understand this antigenic diversity threshold, instead of lumping together all viral strains into a single phenotypic class, viral diversity was introduced by modeling the underlying fitness landscape of HIV-1: different viral strains lead to a different phenotype. The model here developed will be narrowed to a very simple interaction scheme where specific HIV-1 strains interact only with some specific lineage of $\mathrm{CD}^{+} \mathrm{T}$ cells capable of clearing the virus but also being damaged by its infection. The specific nature of the response is introduced in a minimal model with $2 n$ equations (Nowak et al. 1991) defined as:

$$
\begin{aligned}
& \frac{d v_{i}}{d t}=v_{i}\left(r-p x_{i}\right), \\
& \frac{d x_{i}}{d t}=k v_{i}-u v x_{i} .
\end{aligned}
$$

Here $x_{i}$ and $v_{i}$ indicate populations associated to the $i$-th virus strain and its corresponding $\mathrm{CD} 4^{+} \mathrm{T}$ cell partner. For simplicity it is assumed that all viruses replicate with the same rate $r$ and the immune response given by the term $p x_{i} v_{i}$ is also homogeneous. Similarly, the growth and clearance terms in the second equation use identical parameters for all strains. The interactions outlined above are graphically summarized in Fig. 12a-b. Figure 12a displays the network of interactions between the two compartments, including both specific and nonspecific interactions. This diagram is made more explicit in Fig. $12 \mathrm{~b}$ by using one of the pairs of strains $\left(x_{i}\right.$ and $v_{i}$ ) with all the assumed interactions.

Summing over all the previous equations, we obtain the following expressions for the total viral population,

$$
\frac{d v}{d t}=v\left(r-p \sum_{i=1}^{n} x_{i} \frac{v_{i}}{v}\right),
$$

and the corresponding immune cell response:

$$
\sum_{i} \frac{d x_{i}}{d t}=k v-u v \sum_{i} x_{i} .
$$

The virus population will be under control of the immune response, $i$. e. $d v / d t<0$ provided that the following inequality holds:

$$
\frac{r}{p}<\sum_{i=1}^{n} x_{i} \frac{v_{i}}{v}
$$

From the equation for $x_{i}$, we can see that the immune response leads to a stationary state, i. e. $x_{i}=k v_{i} /(u v)$. 

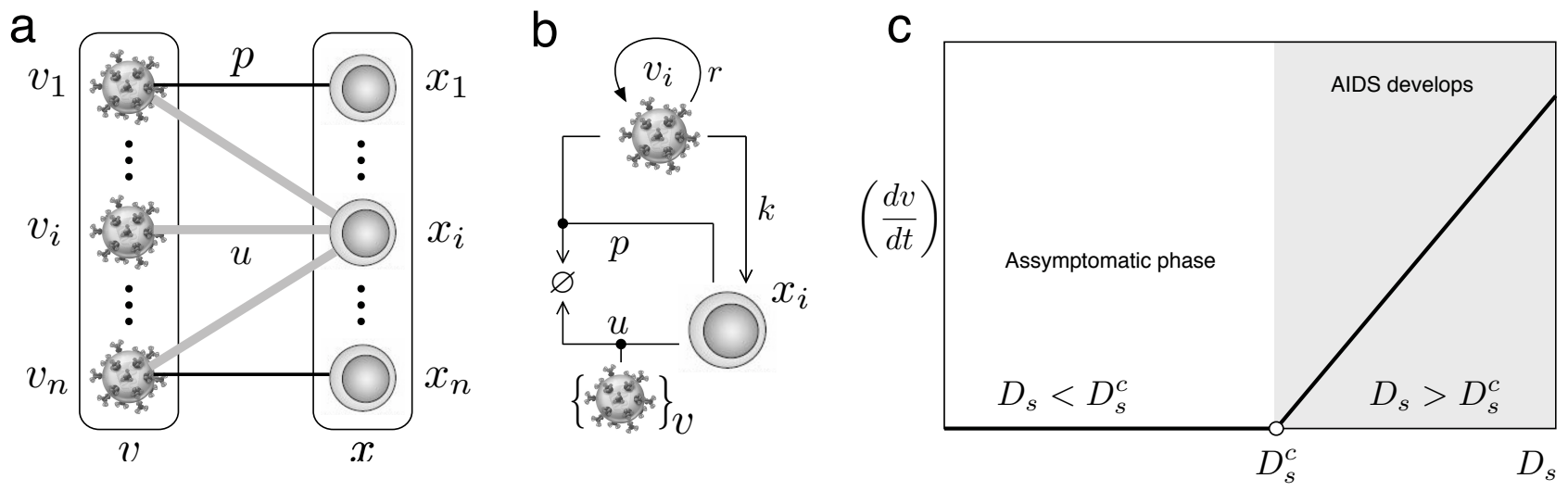

FIG. 12: Antigenic diversity model of HIV-immune system evolution. The model assumes two interacting virus-cell populations involving $n$ strains. Both specific (black lines) and non-specific (gray lines) interactions are possible, revealing the presence of a deep asymmetry. A more detailed graph is shown in (b) where all the basic interactions and their associated parameters are indicated. The set $v$ of all viruses (bottom) indicates that a non-specific interaction is at work. (c) Transition from contained to non-contained (gray area) phase of viral growth.

Using this result and applying it to the total viral population, we obtain an equation for the overall virus population that depends on the actual diversity:

$$
\frac{d v}{d t}=v\left(r-p \frac{k}{u}\left(1-D_{s}\right)\right)
$$

where $D_{s}$ stands for Simpson's diversity index

$$
D_{s}=1-\sum_{i=1}^{n}\left(\frac{v_{i}}{v}\right)^{2}
$$

used in theoretical ecology as a measure of diversity. This measure is maximal for a totally homogeneous population with all strains equally represented, i. e. $D_{s}=1-1 / n$ and minimal when all viruses belong to the same strain, and $D_{s}=0$.

The previous equation for the viral load can be rewritten as follows

$$
\frac{d v}{d t}=\frac{p k}{u} v\left(D_{s}-D_{s}^{c}\right),
$$

with $D_{s}^{c}=1-r u /(p k)$. From this definition, we have a threshold condition separating a phase with no (o very low) viral growth from the escape phase where viral load grows. This is summarized in Fig. 12c, where we schematically indicate the qualitative change from no increasing diversity to the second phase (here there is an evolutionary dynamics over time) highlighted in gray where antigenic diversity starts growing.

This model thus provides an elegant explanation of what is taking place throughout the latency phase in terms of a continuous phase transition. It also allows to understand this transition in terms of a virus-immune system arms race and makes a well-defined prediction. The model suggests (as it seems to be the case) that the long and apparently calm interval of low viremia and slow decay of $\mathrm{CD} 4^{+} \mathrm{T}$ cells hides a rapid turnover of constantly emerging viral escape mutants while a progressive damage to immune responses takes place. This would explain the apparent dormancy of the virus: in reality, there is a constant battle between host defenses and the new variants being created through mutation. This battle keeps the viral load at low levels, but also involves a high cost due to the constant turnover of CD4 ${ }^{+} \mathrm{T}$ cells.

The virus-host dynamics described above has been developed under an extremely simplified model, which lacks several layers of realism. However, a proper implementation using a quasispecies description including mutation rates and other relevant features essentially confirms the presence of the diversity threshold (Nowak and May 1991). Other authors have also addressed the nonlinear dynamics of HIV, showing how simple models can help understanding the tempo and mode of the process (Perelson and Nelson 1999; dos Santos 2001; Funk et al. 2005) and some of them inspired optimization models to maximize the immune response against the virus (Culshaw et al. 2004). Other studies connect the phase transition phenomena described for quasispecies within the context of viruses infecting cells (Alonso and Fort 2010). Finally, some potential connections with phase transitions in percolation theory (Kamp and Bornholdt 2002) have also been put forward.

\section{DISCUSSION}

To build a viral capsid, a concentration threshold of capsomers needs to be surpassed. Afterwards, selfassembly processes take control and allow building a new supramolecular structure required to complete the virus life cycle. The difference between the two regimes (subcritical and super-critical) is not of quantitative nature. 
There are system-level processes that are unleashed in the second phase that have a deep biological meaning that cannot occur in the first phase, where the outcome is disconnected from biological function. The same can be said in a much larger scale, when infection by already well-formed viruses is mediated by intra-host, cell-virus interactions or (at the epidemic spreading level) to the organisms carrying them.

At this levels of description, a novel phenomenon occurs: epidemic spreading. On this scale, the selfassembly example becomes irrelevant as part of the description on the higher scale. However, we have again an interesting mathematical similarity associated to the fundamental nature of the critical condition: success and failure of infection are separated by a marked threshold. Against our intuition, there is no continuous increase in the number of infected agents (either cells or individuals) as infection efficiently increases. Instead, nonlinearities allow or suppress infection. In other examples, such as the multipartite virus dynamics case study or the recombination model, the transition can be sharp, described as a discontinuity. A very small parameter change translates into a catastrophic shift. Either way, a minor changes in key features can end in major changes in the qualitative behavior of the entire system. This is a very important point, well known within epidemiology but perhaps no so well appreciated in other areas.

The consequences of transition thresholds are considerable, since they affect the persistence of multipartite viruses in metapopulation landscape or allow designing ways of enhancing immune responses against retroviruses. The implications are no less relevant. One in particular needs to be highlighted; because of the inevitable occurrence of transition phenomena, punctuated change should be expected to occur frequently. Small changes in parameters, if they cross transition points, trigger qualitative changes with potentially major ecological or functional impacts. Since RNA viruses have high mutation rates, such events are likely to provide a source of innovation. In that case, it would be possible to approach major evolutionary transitions under the umbrella of critical phenomena.

Two important venues will need further theoretical exploration in the future. One is the development of evolutionary theories of change where phase transition points are reached through coevolutionary dynamics. The error catastrophe (Eigen 1971) is a potential example: RNA viruses have coevolved with immune selection barriers to a critical mutation rate. In this view, evolution pushed viral quasispecies to criticality in a self-organized fashion. The evolutionary as well as the ecological dimensions should be integrated to show how particular life forms, such as multipartite viruses, can emerge. Such studies are likely to require the introduction of genomes in some explicit form. Finally, the diverse dynamical and spatial scales considered in each example naturally suggest the minimal requirements for each level and why parameters and features relevant at the lower scale can be ignored. Does it mean they are disconnected? Probably not, and the right answers will need an explicit description of complexity that includes several simultaneous complexity layers.

\section{ACKNOWLEDGMENTS}

This work has been supported by the Botín Foundation by Banco Santander through its Santander Universities Global Division (R.S.), grants FIS2015-67616P (R.S.), RYC-2017-22243 (J.S.), RTI2018-098322-B-I00 (J.S.) and BFU201565037-P (S.F.E) from Spain's Agencia Estatal de Investigación - FEDER, grant PROMETEU/2019/012 from Generalitat Valenciana (S.F.E.), grant DEB-1830699 from the U.S.A. National Science Foundation (S.F.E.), and by the Santa Fe Institute (R.S. and S.F.E.).
Aguirre, J. and Manrubia, S.C. (2008) Effects of spatial competition on the diversity of a quasispecies. Phys. Rev. Lett. 100, 038106 .

Alonso, J. and Fort, H. (2010) Error catastrophe for viruses infecting cells: analysis of the phase transition in terms of error classes. Philosophical Transactions of the Royal Society A 368, 5569-5582.

Altmeyer, S. and McCaskill, J.S.. (2001) Error threshold for spatially resolved evolution in the quasispecies model. Phys. Rev. Lett. 86, 5819.

Anderson RM, May RM (1992) Infectious diseases of humans. Oxford University Press. Oxford: UK.

Balcan D, Vespignani A (2011) Phase transitions in contagion processes mediated by recurrent mobility patterns. Nature Physics 7, 581-586.
Barabási, A.L. (2016) Network science. Cambridge U. Press.

Barthélemy M, Barrat A, Pastor-Satorras R, Vespignani A (2005) Dynamical patterns of epidemic outbreaks in complex heterogeneous networks. Journal of Theoretical Biology 235, 275-288.

Bascompte, J. and Solé, R, (1996) Habitat fragmentation and extinction thresholds in spatially explicit models. Journal of Animal Ecology 65,465-473.

Beekman, M. Sumpter, D.J. and Ratnieks, F.L. (2001) Phase transition between disordered and ordered foraging in Pharaoh's ants. Proceedings of the National Academy of Sciences U.S.A. 98, 9703-9706.

Bocharov, G. Volpert, V. Ludewig, B. and Meyerhans, A. (2018) Mathematical immunology of virus infections. Springer. 
Boerlijst M, Bonhoeffer S, Nowak MA (1996) Viral quasispecies and recombination. Proceedings Royal Society London B 263, 1577-1584.

Bosque G, Folch-Fortuny A, Picó J, Ferrer A, Elena SF (2014) Topology analysis and visualisation of Potyvirus proteinprotein interaction network. BMC Systems Biology 8, 129.

Bull JJ, Meyers LA, Lachmann M (2005) Quasispecies made simple. PLoS Computational Biology 1, e61.

Brockmann, D. and Helbing, D. 2013. The hidden geometry of complex, network-driven contagion phenomena. Science 342 , 1337-1342.

Catalán P, Arias CF, Cuesta JA, Manrubia S (2017) Adaptive multiscapes: an up-to-date metaphor to visualize molecular adaptation. Biology Direct 12: 7 .

Castillo V, Lázaro JT, Sardanyés J (2017) Dynamics and bifurcations in a simple quasispecies model of tumorigenesis. Computational Applied Mathematics 36, 415-431.

Cervera H, Lalić J, Elena SF (2016) Effect of host species on topography of the fitness landscape for a plant RNA virus. Journal of Virology 90, 10160-10169.

Coombs D, Gilchrist MA, Ball CL (2007) Evaluating the importance of within- and between-host selection pressures on the evolution of chronic pathogens. Theoretical Population Biology 72, 576-591.

Christensen, K. and Moloney, N.R. 2005. Complexity and criticality. (Vol. 1). World Scientific Publishing Company. Singapore.

Cuevas JM, Geller R, Garijo R, López-Aldeguer J, Sanjuán R. Extremely high mutation rate of HIV-1 in vivo. PLoS Biology 13(9):e1002251.

Cuevas JM, Durán-Moreno M, Sanjuán R (2017) Multi-virion infectious units arise from free viral particles in an enveloped virus. Nature Microbiology 2, 17078.

Culshaw, R.V. Ruan, S. and Spiteri, R.J. (2004) Optimal HIV treatment by maximising immune response. Journal Mathematical Biology 48, 545-562.

Dezso, Z. and Barabási, A.L. (2002) Halting viruses in scalefree networks. Physical Review E 65, 055103.

dos Santos, R.M.Z. and Coutinho, S. 2001. Dynamics of HIV infection: A cellular automata approach. Physical Review Letters 87, 168102.

Dharmavaram S, Xie F, Klug W, Rudnick J, Bruinsma R (2017) Orientational phase transitions and the assembly of viral capsids. Physical Review E 95, 062402.

Di Collobiano, S.A. Christensen, K. and Jensen, H.J. (2003) The tangled nature model as an evolving quasi-species model. Journal of Physics A 36, 883.

Dill, K. and Bromberg, S. (2012). Molecular driving forces: statistical thermodynamics in biology, chemistry, physics, and nanoscience. Garland Science.

Doekes HM, Fraser C, Lythgoe KA (2017) Effect of the latent reservoir on the evolution of HIV at the within- and betweenhost levels. PLoS Computational Biology 13, e1005228.

Domingo E, Holland JJ, Biebricher C, Eigen M (1995) Quasispecies: the concept and the word. In: Gibbs, A. Calisher, C. Garcia-Arenal, F. (Eds.), Molecular Evolution of the Viruses. Cambridge University Press, Cambridge.
Domingo E, Sheldon J, Perales C (2012) Viral quasispecies evolution. Microbiology Molecular Biology Reviews 76: 159216.

Dorratoltaj N, Nikin-Beers R, Ciupe SM, Eubank SG, Abbas KM (2017) Multi-scale immunoepidemiological modelling of within-host and between-host HIV dynamics systematic review of mathematical models. PeerJ 5: e3877.

Doumayrou J, Avellan A, Froissart R, Michalakis Y (2012) An experimental test of the transmission-virulence trade-off hypothesis in a plant virus. Evolution 67: 477-486.

Eigen M (1971) Selforganisation of matter and evolution of biological macromolecules. Naturwissenschaften 58, 465-523.

Eigen M, McCaskill J, Schuster P (1988) Molecular quasispecies. Journal Physical Chemistry 92(24), 6881-689.

Eigen M, McCaskill J, Schuster P (1989) The molecular quasispecies. Advances Chemical Physics 75, 149-263.

Elena SF, Solé RV, Sardanyés J (2010) Simple genomes, complex interactions: Epistasis in RNA virus. Chaos 20, 026106.

Feng Z, Velasco-Hernández J, Tapia-Santos B, Leite MCA (2012) A model for coupling within-host and between-host dynamics in an infectious disease. Nonlinear Dynamics 68: 401-411.

Funk, G.A. Jansen, V.A. Bonhoeffer, S. and Killingback, T. (2005) Spatial models of virus-immune dynamics. Journal of Theoretical Biology 233, 221-236.

Fontich E, Sardanyés J. (2008) General scaling law in the saddle-node bifurcation: a complex phase space study?. Journal of Physics A: Mathematical and Theoretical 41, 1-9.

Freitas RA, Merkle RC. (2004) Kinematic self-replicating machines. Landes Bioscience. Georgetown.

Gago S, Elena SF, Flores R, Sanjuán R (2009). Extremely High Mutation Rate of a Hammerhead Viroid. Science 323: 1308-1308.

Hufnagel, L. Brockmann, D. and Geisel, T. 2004. Forecast and control of epidemics in a globalized world. Proceedings of the National Academy of Sciences U.S.A. 101, 15124-15129.

Kamp, C. and Bornholdt, S. (2002) From HIV infection to AIDS: a dynamically induced percolation transition?. Proceedings of the Royal Society B 269, 2035-2040.

Kitsak, M. Gallos, L.K. Havlin, S. Liljeros, F. Muchnik, L. Stanley, H.E. and Makse, H.A. (2010) Identification of influential spreaders in complex networks. Nature Physics 6, 888-893.

Goldenfeld, N. 2018. Lectures on phase transitions and the renormalization group. CRC Press.

Gong JR, Pellis L, Wood JLN, McLean AR, Arinaminpathy N, Lloyd-Smith JO (2015) Seven challenges in modelling pathogen dynamics within-host and across scales. Epidemics 10: $45-48$.

Hagan, M.F. and Chandler, D. 2006. Dynamic pathways for viral capsid assembly. Biophysical Journal, 9, 42-54.

Hagan MF (2014) Modelling viral capsid assembly. Adv Chem Phys 155, 1-68.

Haken, H. 1975. Cooperative phenomena in systems far from thermal equilibrium and in nonphysical systems. Reviews of Modern Physics, 47, 67-121. 
Hanski, I. 1999. Metapopulation ecology. Oxford University Press.

Heldt FS, Fresing T, Pflugmacher A, Gröpler R, Peschel B, Reichl, U (2013) Multiscale modelling of Influenza A virus infection supports the development of direct-acting antivirals. PLoS Computational Biology 9: e1003372.

Hicks, S.D. and Henley, C.L. 2006. Irreversible growth model for virus capsid assembly. Physical Review E, 74, 031912.

Iranzo J, Manrubia S (2012) Evolutionary dynamics of genome segmentation in multipartite viruses. Proceedings of the Royal Society B 279, 3812-3819.

Jaeger, G (1998) The Ehrenfest classification of phase transitions: introduction and evolution". Archive for History of Exact Sciences 53, 51-81.

Kadanoff, L.P. 2000. Statistical physics: statics, dynamics and renormalization. World Scientific Publishing Company. Singapore.

Klug, A. (2010) From virus structure to chromatin: X-ray diffraction to three-dimensional electron microscopy. Annual Review of Biochemistry 79, 1-35.

Korenromp EL, Williams BG, Schmid GP, Dye C (2009) Clinical prognostic value of RNA viral load and CD4 cell counts during untreated HIV-1 infection ? a quantitative review. PLoS ONE 4: e5950.

Kouyos RD, Leventhal GE, Hinkley T, Haddad M, Whitcomb $\mathrm{JM}$, et al. (2012) Exploring the complexity of the HIV-1 fitness landscape. PLoS Genetics 8, e1002551.

Kumberger P, Frey F, Schwarz US, Graw F (2016) Multiscale modelling of virus replication and spread. FEBS Letters 590, 1972-1986.

Lalić J, Elena SF (2015) The impact of high-order epistasis in the within-host fitness of a positive-sense plant RNA virus. Journal of Evolutionary Biology 28:,2236-2247.

Lemey P, Rambaut A, Pybus OG (2006) HIV evolutionary dynamics within and among hosts. AIDS Reviews 8: 125-140.

Leuthäusser, I. (1986) An exact correspondence between Eigen's evolution model and a two-dimensional Ising system. Journal of Chemical Physics 84, 1884-1885.

Leuthäusser, I. (1987) Statistical mechanics of Eigen's evolution model. Journal of Statistical Physics 48, 343-360.

Liu R, Li M, Liu ZP, Wu J, Chen L, et al. (2012) Identifying critical transitions and their leading biomolecular networks in complex diseases. Scientific Reports 2, 813.

Lloyd, A.L. and May, R.M. (2001) How viruses spread among computers and people. Science 292, 1316-1317.

Loverdo C, Park M, Schreiber SJ, Lloyd-Smith JO (2012) Influence of viral replication mechanisms on within-host evolutionary dynamics. Evolution 66, 3462-3471.

Lucía-Sanz, A. and Manrubia, S. (2017) Multipartite viruses: adaptive trick or evolutionary treat?. NPJ systems biology and applications, 3, 1-11.

Lucía-Sanz A, Aguirre J, Manrubia S (2018) Theoretical approaches to disclosing the emergence and adaptive advantages of multipartite viruses. Current Opinion in Virology 33, 89-95.
Metzger VT, Lloyd-Smith JO, Weinberger LS (2011) Autonomous targeting of infectious superspreaders using engineered transmissible therapies. PLoS Computational Biology 7, e1002015.

Mideo N, Alizon S, Day T (2008) Linking within- and between-host dynamics in the evolutionary epidemiology of infectious diseases. Trends Ecology and Evolution 23, 511517.

Moilanen, A. and Hanski, I. 1998. Metapopulation dynamics: effects of habitat quality and landscape structure. Ecology 79, 2503-2515.

Mouritsen O. (2005) Life-as a matter of fat: the emerging science of lipidomics. Berlin: Springer.

Mustonen V, Lässig M (2009) From fitness landscapes to seascapes: non-equilibrium dynamics of selection and adaptation. Trends in Genetics 25, 111-119.

Nicolis, G. and Nicolis, G. 1995. Introduction to nonlinear science. Cambridge University Press.

Nowak, M.A. and May, R.M. (1991) Mathematical biology of HIV infections: antigenic variation and diversity threshold. Math. Biosci. 106, 1-21.

Nowak MA, Anderson RM, Boerlijst MC, Bonhoeffer S, May RM, McMichael, AJ (1996) HIV-1 evolution and disease progression. Science 274: 1008-1011.

Nowak MA, Anderson RM, McLean AR, Wolfs TF, Goudsmit J, May RM (1991) Antigenic diversity thresholds and the development of AIDS. Science 254, 963-969.

Nowak, M. and May, R.M. (2000) Virus dynamics: mathematical principles of immunology and virology: mathematical principles of immunology and virology. Oxford University Press, UK.

Nurtay A. Hennessy MG. Sardanyés J. Alsedà Ll. Elena, SF (2019) Theoretical conditions for the coexistence of viral strains with differences in phenotypic traits: a bifurcation analysis. Royal Society Open Sciences 6, 181179

Park, J.M. and Deem, M.W. (2007) Phase diagrams of quasispecies theory with recombination and horizontal gene transfer. Physical Review Letters 98, 058101.

Park M, Loverdo C, Schreiber SJ, Lloyd-Smith JO (2013) Multiple scales of selection influence the evolutionary emergence of novel pathogens. Philosophical Transactions of the Royal Society B 368, 20120333.

Pastor-Satorras, R. and Vespignani, A. (2001) Epidemic spreading in scale-free networks. Physical Review Letters 86, 3200 .

Pastor-Satorras, R. and Solé, R. (2001). Field theory for a reaction-diffusion model of quasispecies dynamics. Physical Reviews E 64, 051909.

Pastor-Satorras, R. Castellano, C. Van Mieghem, P. and Vespignani, A. (2015) Epidemic processes in complex networks. Reviews Modern Physics 87, 925.

Perelson, A.S. and Weisbuch, G. (1997) Immunology for physicists. Reviews of Modern Physics 69, 1219.

Perelson, A.S. and Nelson, P.W. (1999) Mathematical analysis of HIV-1 dynamics in vivo. SIAM Review, 41, 3-44.

Perelson, A.S. 2002. Modelling viral and immune system dynamics. Nature Reviews Immnunology 2, 28-36. 
Perlmutter, J.D. and Hagan, M.F. (2015) Mechanisms of virus assembly. Annual Review of Physical Chemistry 66, 217-239.

Perlmutter, J.D. Qiao, C. and Hagan, M.F. (2013) Viral genome structures are optimal for capsid assembly. eLife, 2, $\mathrm{e} 00632$.

Piñero, J. and Solé, R. (2019). Statistical physics of liquid brains. Philosophical Transactions Royal Society B 374, 20180376.

Rapaport, D.C. (2010). modelling capsid self-assembly: design and analysis. Physical Biology 7(4), 045001.

Rapaport, D.C. (2014). Molecular dynamics simulation: a tool for exploration and discovery using simple models. Journal Physics Condensed Matter. 26, 503104.

Saakian, D.B. Munoz, E. Hu, C.K. and Deem, M.W. (2006) Quasispecies theory for multiple-peak fitness landscapes. Physical Review E 73, 041913.

Saakian, D.B. 2018. Quasispecies model of evolution with migration. Physical Review E 98, 012414.

Sardanyés J (2009) Dynamics, evolution and information in nonlinear dynamical systems of replicators ( $\mathrm{PhD}$ diss. Universitat Pompeu Fabra), Barcelona.

Sardanyés J, Solé RV, Elena SF (2009) Replication mode and landscape topology differentially affect RNA virus mutational load and robustness. Journal of Virology 83, 12579-12589.

Sardanyés J, Elena SF (2011) Quasispecies spatial models for RNA virus with different replication modes and infection strategies. PLoS ONE 6, e24884.

Sardanyés J, Martínez R, Simó C, Solé R (2017) Abrupt transitions to tumor extinction: A phenotypic quasispecies model. Journal of Mathematical Biology 74, 1589-1609.

Sardanyés J, Simó C, Martínez R (2018) Trans-heteroclinic bifurcation: A novel type of catastrophic shift. Royal Society Open Science 5, 171304.

Sardanyés J, Alarcón T (2018) Noise-induced bistability in the fate of cancer phenotypic quasispcies: a bit-strings approach. Scientific Reports 8, 1027.

Sardanyés, J. Piñero, J. and Solé, R. (2019) Habitat lossinduced tipping points in metapopulations with facilitation. Population Ecology 61, 436-449.

Scholle SO, Ypma RJF, Lloyd AL, Koelle K (2013) Viral substitution rate variation can arise from the interplay between within-host and epidemiological dynamics. American Naturalist 182: 494-513.

Shin J, MacCarthy T (2016) Potential for evolution of complex defense strategies in a multi-scale model of virus-host coevolution. BMC Evolutionary Biology 16: 233.

Sicard A, Michalakis Y, Gutiérrez S, Blanc S (2016) The strange lifestyle of multipartite viruses. PLoS Pathogens. 12, e1005819.

Sicard A, Pirolles E, Gallet R, Vernerey MS, Yvon M, Urbino C, Peterschmitt M, Gutiérrez S, Michalakis Y, Blanc, S (2019) A multipartite way of life for a multipartite virus. eLife 8 , e43599.
Solé, R and Goodwin, B. C. (2001) Signs of Life: How complexity pervades biology. Basic Books, New York.

Solé, R. (2003) Phase transitions in unstable cancer cell populations. European Physics Journal B 35, 117-123.

Solé, R. and Deisboeck, T.S. (2004) An error catastrophe in cancer? Journal of Theoretical Biology 228, 47-54.

Solé R, Sardanyés J, Diez J, Mas A (2006) Information catastrophe in RNA viruses through replication thresholds. Journal of Theoretical Biology 240, 353-359

Solé, R. (2009) Evolution and self-assembly of protocells. Intl. J. Biochem. Cell Biology 41, 274-284.

Solé, R. (2011) Phase Transitions. Princeton University Press. Princeton: USA.

Solé, R. (2012). Phase transitions in cancer. In : D'onofrio, A., Cerrai, P. and Gandolfi, A. (eds.) New Challenges for Cancer Systems Biomedicine (pp. 35-51). Springer, Milano.

Solé R, Elena SF (2019) Virus as Complex Adaptive Systems. Princeton University Press. Princeton: USA.

Stanley, H.E. Amaral, L.A. Buldyrev, S.V. et al. (1996) Scaling and universality in animate and inanimate systems. Physica A 231, 20-48.

Stich, M., Briones, C. and Manrubia, S.C. (2007). Collective properties of evolving molecular quasispecies. BMC Evolutionary Biology 7, 110.

Stollenwerk, N. and Jansen, V. (2011) Population Biology and Criticality: From critical birth-death processes to selforganized criticality in mutation pathogen systems. World Scientific.

Strogatz, S., (1994) Nonlinear dynamics and chaos: with applications to physics, biology, chemistry, and engineering. Addison-Wesley, Reading, MA.

Swetina J, Schuster P (1982) Self-replication with errors. A model for polynucleotide replication. Biophysal Chemistry 16, 329

Tarazona, P. 1992. Error thresholds for molecular quasispecies as phase transitions: From simple landscapes to spin-glass models. Physical Review A 45, 6038.

Valdano, E., Manrubia, S., Gómez, S. and Arenas, A. (2019) Endemicity and prevalence of multipartite viruses under heterogeneous between-host transmission. PLoS Computational Biology 15, e1006876.

Wang, Z. Bauch, C.T. Bhattacharyya, S. et al. (2016) Statistical physics of vaccination. Physics Reports 664,1-113.

Weissmann, H. and Shnerb, N.M. (2014) Stochastic desertification. Europhysics Letters 106(2), 28004.

Whitesides, G.M. and Grzybowski, B. (2002) Self-assembly at all scales. Science 295, 2418-2421.

Wodarz, D. (2007). Killer cell dynamics Springer, New York.

Zlotnick, A. (2004) Viruses and the physics of soft condensed matter. Proceedings of the National Academy of Sciences U.S.A. 101, 5549-15550. 